Operator Theory:

Advances and Applications, Vol. 1, 1-37

(c) 2021 Birkhäuser Verlag Basel/Switzerland

\title{
The Schur Transformation for Nevanlinna Functions: Operator Representations, Resolvent Matrices, and Orthogonal Polynomials
}

\author{
D. Alpay, A. Dijksma and H. Langer \\ Dedicated to Mark Krein on the occasion of his 100th anniversary
}

\begin{abstract}
A Nevanlinna function is a function which is analytic in the open upper half plane and has a non-negative imaginary part there. In this paper we study a fractional linear transformation for a Nevanlinna function $n$ with a suitable asymptotic expansion at $\infty$, that is an analogue of the Schur transformation for contractive analytic functions in the unit disc. Applying the transformation $p$ times we find a Nevanlinna function $n_{p}$ which is a fractional linear transformation of the given function $n$. The main results concern the effect of this transformation to the realizations of $n$ and $n_{p}$, by which we mean their representations through resolvents of self-adjoint operators in Hilbert space. Our tools are block operator matrix representations, $u$-resolvent matrices, and reproducing kernel Hilbert spaces.
\end{abstract}

Mathematics Subject Classification (2000). Primary 47A57, 47B32, Secondary 42C05, 30E05.

Keywords. Schur transformation, Nevanlinna function, realization, symmetric operator, self-adjoint operator, moment problem, reproducing kernel Hilbert space, orthogonal polynomials.

D. Alpay acknowledges with thanks the Earl Katz family for endowing the chair which supported this research. The research of A. Dijksma and H. Langer was supported in part by the Center for Advanced Studies in Mathematics (CASM) of the Department of Mathematics, Ben-Gurion University. 


\section{Introduction}

In the papers [4] and [5] the Schur transformation for generalized Nevanlinna functions with a reference point $z_{1}$ in the open upper half plane was considered. An analogous transformation for Nevanlinna functions (for the definition of a Nevanlinna function see Section 2) and for the reference point $\infty$ is defined in [1, Lemma 3.3.6], see 3. This transformation or a simple modification of it we call here the Schur transformation for Nevanlinna functions, and it is the starting point for the present paper. To give more details, we consider a Nevanlinna function $n$ which has for some integer $p \geq 1$ an asymptotic expansion of order $2 p+1$ at $\infty$, for example

$$
n(z)=-\frac{s_{0}}{z}-\frac{s_{1}}{z^{2}}-\cdots-\frac{s_{2 p}}{z^{2 p+1}}+\mathrm{o}\left(\frac{1}{z^{2 p+1}}\right), \quad z=\mathrm{i} y, y \rightarrow \pm \infty .
$$

The Schur transform $\widehat{n}$ of $n$ is the function

$$
\widehat{n}(z):=-\frac{s_{0}}{n(z)}-z+\frac{s_{1}}{s_{0}}
$$

the relation between $n$ and $\widehat{n}$ can also be written as

$$
n(z)=-\frac{s_{0}}{z-\frac{s_{1}}{s_{0}}+\widehat{n}(z)} .
$$

The transformed function $\widehat{n}=: n_{1}$ is again a Nevanlinna function, but in general with an asymptotic expansion of the form (1.1) of lower order $2 p-1$, and if $p>1$ the Schur transformation can be again applied to $n_{1}$ etc. As a result we obtain a finite sequence of Nevanlinna functions $n_{1}=\widehat{n}, n_{2}=\widehat{n}_{1}, \ldots, n_{p}=\widehat{n}_{p-1}$; this is the sequence of functions that appears in the asymptotic expansion of $n$ by continued fractions, see [1, Section 3.3.6].

The transformation (1.2) is closely related to the finite Hamburger moment problem. We recall that the Nevanlinna function $n$ with an asymptotic expansion (1.1) admits an integral representation

$$
n(z)=\int_{-\infty}^{\infty} \frac{d \sigma(t)}{t-z}, \quad z \neq z^{*}
$$

where $\sigma$ is a bounded non-decreasing function on $\mathbb{R}$. The coefficients $s_{j}$ in (1.1) are the moments of the function $\sigma$ :

$$
s_{j}=\int_{-\infty}^{\infty} t^{j} d \sigma(t), \quad j=0,1, \ldots, 2 p .
$$

The moment problem we have in mind is the problem to determine all Nevanlinna functions $n$ with an expansion (1.1) and given coefficients $s_{j}, j=0,1, \ldots, p$, see [1, Theorem 3.2.1].

An essential feature in our studies are operator representations or so-called realizations of Nevanlinna functions, see [13, [10, and [9]. In fact, if the Nevanlinna 
function $n$ admits an asymptotic expansion (1.1) its operator representation takes the simple form

$$
n(z)=\left((A-z)^{-1} u, u\right), \quad z \neq z^{*}
$$

with some Hilbert space $\mathcal{H}$ with inner product $(\cdot, \cdot), u \in \mathcal{H}$, and a self-adjoint operator $A$ in $\mathcal{H}$. We study the corresponding operator representation of the Schur transform $\widehat{n}$, and also of the functions $n_{2}, \ldots, n_{p}$. For example, the function $\widehat{n}$ admits an operator representation of the form (1.4) with a Hilbert space $\widehat{\mathcal{H}}$, an operator $\widehat{A}$, and an element $\widehat{u}$ which are the orthogonal complement of the element $u$ in $\mathcal{H}$, the compression of $A$ to $\widehat{\mathcal{H}}$, and a multiple of the projection of $A u$ onto $\widehat{\mathcal{H}}$, respectively. After applying the Schur transformation $p$ times, the resulting function $n_{p}$ admits an operator representation of the form (1.4) with the space

$$
\mathcal{H}_{p}^{\prime}=\mathcal{H} \ominus \mathcal{H}_{p}, \quad \mathcal{H}_{p}:=\operatorname{span}\left\{u, A u, \ldots, A^{p-1} u\right\},
$$

the operator that is the compression of $A$ to this space, and an element which is a multiple of the projection of $A^{p} u$ onto $\mathcal{H}_{p}^{\prime}$.

Since $n_{p}$ is obtained by subsequent application of fractional linear transformations of the form (1.2), there is a fractional linear relation between the function $n$ and the transformed function $n_{p}$. We derive an explicit form for the defining $2 \times 2$ matrix function $V$ of this relation in three ways: By calculating the resolvent of the operator $A$ in its $2 \times 2$ block matrix operator form corresponding to the decomposition $\mathcal{H}=\mathcal{H}_{p} \oplus \mathcal{H}_{p}^{\prime}$, by means of the description of all generalized resolvents of a certain symmetric restriction of $A$ with defect one in the space $\mathcal{H}_{p+1}$, and via reproducing kernel methods using the non-negative Nevanlinna kernel

$$
L_{n}(z, w)=\frac{n(z)-n(w)^{*}}{z-w^{*}}, \quad z, w \in \mathbb{C} \backslash \mathbb{R}, z \neq w^{*} .
$$

For the Nevanlinna function $n$ with an asymptotic expansion (1.1), polynomials $e_{j}$ and $d_{j}, j=1,2, \ldots, p$, of first and second kind can be defined by the well-known formulas, see [1, Chapter I]. Recall that $e_{j}$ is a polynomial of degree $j$, and that $d_{j}$ is a polynomial of degree $j-1$. We show that the polynomials $\widehat{e}_{j}$ of first kind of the transformed function $\widehat{n}$ coincide, up to constant factor, with the polynomials $d_{j+1}(z)$ of second kind for the given function $n$, whereas the polynomials $\widehat{d}_{j}$ of second kind for $\widehat{n}$ are linear combinations of $e_{j+1}$ and $d_{j+1}$. As a consequence, the polynomials of second kind for $n$ are orthogonal with respect to the measure generated by the non-decreasing function $\widehat{\sigma}$ in the representation of the form (1.4) of the Nevanlinna function $\widehat{n}$; in this statement $\widehat{n}$ can be replaced by the function $-1 / n$. As in the classical moment problem, the $2 \times 2$ matrix function $V$, which determines the fractional linear relation between $n$ and $n_{p}$, can be represented by the polynomials of first and second kind.

A short synopsis is as follows. The Schur transformation is defined in the next section. We start with weaker forms of the asymptotic expansion (1.1), for example

$$
n(z)=-\frac{s_{0}}{z}-\frac{s_{1}}{z^{2}}+\mathrm{o}\left(\frac{1}{z^{2}}\right), \quad z=\mathrm{i} y, y \rightarrow \pm \infty,
$$


and consider also a weaker form of the Schur transformation. In Section 3 we mention three concrete forms of the operator representation of $n$. The basic result of this section is Theorem 3.1 which describes the operator model for the transformed function. Higher order approximations and the corresponding polynomials of first and second kind are introduced in Section 4 . In the operator model an asymptotic expansion (1.1) can be characterized by the fact that $u \in \operatorname{dom} A^{p}$. The main result of this section is the relation between the polynomials of first and second kind of $n$ and $\widehat{n}$ which was mentioned above. The reduction via a $p$-dimensional subspace, that corresponds to $p$ subsequent applications of the Schur transformation, is given in Section 5 by means of a block operator matrix representation of $A$. In Section 6 the corresponding transformation matrix $V$ is expressed in terms of the polynomials of first and second kind. Although the final formulas are well known (see for example [1]) this approach seems to be new.

In Section 7, applying the theory of $u$-resolvent matrices, we derive a representation of a transformation matrix in an explicit form by means of the given moments; it corresponds to Potapov's formula for the solution matrix of the Nevanlinna - Pick problem, compare also [2. Finally, in Section 8 we explain the connection between $n$ and $n_{p}$ through some basic results from the theory of resolvent invariant reproducing kernel spaces, and give another proof for the representation of the transformation matrix by orthogonal polynomials.

\section{The Schur transformation}

1. A Nevanlinna function is a complex function $n$ which is defined and analytic in the upper half plane $\mathbb{C}^{+}$and has the property

$$
z \in \mathbb{C}^{+} \Longrightarrow \operatorname{Im} n(z) \geq 0 .
$$

We always suppose that $n$ is extended to the lower half plane $\mathbb{C}^{-}$by the relation

$$
n(z)=n\left(z^{*}\right)^{*}, \quad z \in \mathbb{C}^{-},
$$

and to those points of the real axis into which it can be continued analytically. The set of all Nevanlinna functions is denoted by $\mathbf{N}_{0}$. Recall that $n \in \mathbf{N}_{0}$ if and only if $n$ is analytic in $\mathbb{C} \backslash \mathbb{R}$ and the kernel

$$
L_{n}(z, w)=\frac{n(z)-n(w)^{*}}{z-w^{*}}, \quad z, w \in \mathbb{C} \backslash \mathbb{R}, z \neq w^{*},
$$

is positive definite.

Let $n \in \mathbf{N}_{0}$ and consider the following properties of $n$ :

(1 $\left.1_{0}\right) n(z)=-\frac{s_{0}}{z}+\mathrm{o}\left(\frac{1}{z}\right)$,

$\left(2_{0}\right) n(z)=-\frac{s_{0}}{z}+\mathrm{O}\left(\frac{1}{z^{2}}\right)$, 
$\left(3_{0}\right) n(z)=-\frac{s_{0}}{z}-\frac{s_{1}}{z^{2}}+\mathrm{o}\left(\frac{1}{z^{2}}\right)$,

where here and in the following, the limit relations are understood to hold for $z \rightarrow \pm \infty$ along the imaginary axis. The assumption (2.1) implies that $s_{0}$ and $s_{1}$ are real numbers. Evidently, $\left(3_{0}\right) \Longrightarrow\left(2_{0}\right) \Longrightarrow\left(1_{0}\right)$. The function $n$ satisfies the assumption $\left(1_{0}\right)$ if and only if it belongs to the class $\left(R_{0}\right)$ of [12, which means that it admits an integral representation

$$
n(z)=\int_{-\infty}^{+\infty} \frac{1}{t-z} d \sigma(t), \quad z \in \mathbb{C} \backslash \mathbb{R},
$$

where $\sigma$ is a bounded non-decreasing function on $\mathbb{R}$. Then

$$
\int_{-\infty}^{+\infty} d \sigma(t)=s_{0}
$$

hence $s_{0} \geq 0$, and if $s_{0}=0$ then $n(z) \equiv 0$. With the representation (2.2) of $n$ the assumption

$$
\int_{-\infty}^{\infty}|t| d \sigma(t)<\infty
$$

implies that $\left(3_{0}\right)$ is satisfied. Indeed, (2.3) implies that

$$
s_{1}=\int_{-\infty}^{\infty} t d \sigma(t)
$$

exists and with $z=i y$

$$
z^{2}\left(n(z)+\frac{s_{0}}{z}+\frac{s_{1}}{z^{2}}\right)=\int_{-\infty}^{\infty} \frac{t^{2}}{t-z} d \sigma(t)=\int_{-\infty}^{\infty} \frac{t^{2}+i y t}{t^{2}+y^{2}} t d \sigma(t)=\mathrm{o}(1) .
$$

The assumptions $\left(1_{0}\right),\left(2_{0}\right)$, and $\left(3_{0}\right)$ are all different. To see that $\left(1_{0}\right) \not\left(2_{0}\right)$ we show that if $n \in \mathbf{N}_{0}$ has the representation (2.2) with $\operatorname{supp} \sigma \subset[0, \infty)$ and

$$
\int_{0}^{\infty} d \sigma(t)<\infty, \quad \int_{0}^{\infty} t d \sigma(t)=\infty
$$

then $\left(2_{0}\right)$ does not hold: Let $c>0$ be given arbitrarily (large) and choose $K>0$ such that $\int_{0}^{K} t d \sigma(t) \geq c$. If $y$ is chosen large enough then for $0 \leq t \leq K$ we have

$$
\frac{y^{2}}{t^{2}+y^{2}} \geq \frac{1}{2}
$$

and hence

$$
\int_{-\infty}^{\infty} \frac{y^{2} t}{t^{2}+y^{2}} d \sigma(t) \geq \frac{c}{2}
$$

and therefore, with $z=i y$,

$$
z^{2}\left(n(z)+\frac{s_{0}}{z}\right)=z \int_{-\infty}^{\infty} \frac{t}{t-z} d \sigma(t)=\int_{-\infty}^{\infty} \frac{-y^{2} t+i y t^{2}}{t^{2}+y^{2}} d \sigma(t) \neq \mathrm{O}(1)
$$


which implies that $\left(2_{0}\right)$ does not hold. Thus, for example, the function

$$
n(z)=\frac{-1}{z-\sqrt{-z}}=\int_{0}^{\infty} \frac{1}{t-z} \frac{d t}{\pi(t+1) \sqrt{t}}
$$

satisfies $\left(1_{0}\right)$ but not $\left(2_{0}\right)$.

Let $n$ be the Nevanlinna function, defined in the upper half plane by

$$
n(z)=\frac{-s_{0}}{z+\gamma+f(z)}, z \in \mathbb{C}^{+}
$$

where $s_{0}$ is a positive real number, $\gamma$ is a complex number with $\operatorname{Im} \gamma>0$, and $f$ is a Nevanlinna function such that $f(z)=\mathrm{o}(1)$. It has the properties

$$
\lim _{z=i y, y \rightarrow \infty} z^{2}\left(n(z)+\frac{s_{0}}{z}\right)=\gamma s_{0}, \quad \lim _{z=i y, y \rightarrow-\infty} z^{2}\left(n(z)+\frac{s_{0}}{z}\right)=\gamma^{*} s_{0},
$$

and hence $n$ satisfies $\left(2_{0}\right)$ but, since the two limits are different (and non-real), it does not satisfy $\left(3_{0}\right)$.

Instead of the assumption $\left(3_{0}\right)$ also the assumption

$$
n(z)=-\frac{s_{0}}{z}-\frac{s_{1}}{z^{2}}+\mathrm{O}\left(\frac{1}{z^{3}}\right)
$$

seems reasonable. However, according to [13, Bemerkung 1.11], (2.4) implies the existence of a real number $s_{2}$ such that

$$
n(z)=-\frac{s_{0}}{z}-\frac{s_{1}}{z^{2}}-\frac{s_{2}}{z^{3}}+\mathrm{o}\left(\frac{1}{z^{3}}\right) ;
$$

this relation will be considered in Section 4 as assumption $\left(1_{1}\right)$. The implication (2.4) $\Longrightarrow$ (2.5) can also be seen from the integral representation (2.2) of $n$ : (2.4) implies

$$
z^{3}\left(\int_{-\infty}^{\infty} \frac{1}{t-z}+\frac{1}{z} d \sigma(t)+\frac{s_{1}}{z^{2}}\right)=\mathrm{O}(1)
$$

and hence with $z=i y$

$$
-\int_{-\infty}^{\infty} \frac{y^{2} t(t+i y)}{t^{2}+y^{2}} d \sigma(t)+i y s_{1}=\mathrm{O}(1), \quad y \rightarrow \infty .
$$

Taking the imaginary part we see that

$$
s_{1}=\lim _{y \rightarrow \infty} \int_{-\infty}^{\infty} \frac{y^{2} t}{t^{2}+y^{2}} d \sigma(t)
$$

and taking the real part we see that there exist real numbers $C$ and $y_{0}$ such that

$$
\int_{-\infty}^{\infty} \frac{y^{2} t^{2}}{t^{2}+y^{2}} d \sigma(t) \leq C, \quad y \geq y_{0}
$$

This implies that

$$
s_{2}:=\int_{-\infty}^{\infty} t^{2} d \sigma(t)<\infty
$$


hence

$$
\int_{-\infty}^{\infty}|t| d \sigma(t)<\infty
$$

and

$$
s_{1}=\int_{-\infty}^{\infty} t d \sigma(t) .
$$

Now (2.5) easily follows from the integral representations of $n$ and the expressions for the real numbers $s_{0}, s_{1}$, and $s_{2}$ : With $z=i y$ we have

$$
z^{3}\left(n(z)+\frac{s_{0}}{z}+\frac{s_{1}}{z^{2}}+\frac{s_{2}}{z^{3}}\right)=\int_{-\infty}^{\infty} \frac{t^{3}}{t-z} d \sigma(t)=\int_{-\infty}^{\infty} \frac{t^{4}+i t^{3} y}{t^{2}+y^{2}} d \sigma(t)=\mathrm{o}(1) .
$$

2. Now we define the basic transformations considered this paper.

Definition 2.1. If $n \in \mathbb{N}_{0}$ satisfies the assumption $\left(1_{0}\right)$ or $\left(2_{0}\right)$, the Schur type transform $\tilde{n}$ of $n$ is the function

$$
\widetilde{n}(z)=\frac{-s_{0}}{n(z)}-z
$$

if $n \in \mathbb{N}_{0}$ satisfies the assumption $\left(3_{0}\right)$ the Schur transform $\widehat{n}$ of $n$ is the function

$$
\widehat{n}(z)=\frac{-s_{0}}{n(z)}-z+\frac{s_{1}}{s_{0}} .
$$

The difference between the formulas (2.6) and (2.7) is just in the additive real constant $s_{1} / s_{0}$ : under the stronger assumption $\left(3_{0}\right)$ this constant assures that the transform tends to zero if $z$ tends to $\pm \infty$ along the imaginary axis, see (2.10) below.

The relations (2.6) and (2.7) can also be written as a first step of a continued fraction expansion

$$
n(z)=-\frac{s_{0}}{z+\widetilde{n}(z)}, \quad \text { or } n(z)=-\frac{s_{0}}{z-\frac{s_{1}}{s_{0}}+\widehat{n}(z)} .
$$

Theorem 2.2. The following equivalences hold:

$$
\begin{aligned}
& n \in \mathbf{N}_{0} \text { and satisfies }\left(1_{0}\right) \Longleftrightarrow \widetilde{n} \in \mathbf{N}_{0}, \widetilde{n}(z)=\mathrm{o}(z), \\
& n \in \mathbf{N}_{0} \text { and satisfies }\left(2_{0}\right) \Longleftrightarrow \widetilde{n} \in \mathbf{N}_{0}, \widetilde{n}(z)=\mathrm{O}(1), \\
& n \in \mathbf{N}_{0} \text { and satisfies }\left(3_{0}\right) \Longleftrightarrow \widehat{n} \in \mathbf{N}_{0}, \widehat{n}(z)=\mathrm{o}(1) .
\end{aligned}
$$

Proof. We have

$$
\widetilde{n}(z)=\widehat{n}(z)-\frac{s_{1}}{s_{0}}=-\frac{s_{0}+z n(z)}{n(z)} .
$$

A straightforward calculation yields

$$
\operatorname{Im} \widetilde{n}(z)=\operatorname{Im} \widehat{n}(z)=\frac{\operatorname{Im} z}{|n(z)|^{2}}\left(s_{0} \frac{\operatorname{Im} n(z)}{\operatorname{Im} z}-|n(z)|^{2}\right),
$$

and the estimate

$$
|n(z)|^{2}=\left|\int_{-\infty}^{+\infty} \frac{d \sigma(t)}{t-z}\right|^{2} \leq \int_{-\infty}^{+\infty} \frac{d \sigma(t)}{|t-z|^{2}} \int_{-\infty}^{+\infty} d \sigma(t)=\frac{\operatorname{Im} n(z)}{\operatorname{Im} z} s_{0}
$$


implies $\widetilde{n}, \widehat{n} \in \mathbf{N}_{0}$. The asymptotic properties of $\widetilde{n}$ follow in case (2.8) from the relation

$$
\frac{\widetilde{n}(z)}{z}=-\frac{s_{0}}{z n(z)}-1=\mathrm{o}(1)
$$

in case (2.9) from the relation

$$
\widetilde{n}(z)=-\frac{s_{0}+z n(z)}{n(z)}=\frac{z \mathrm{O}\left(\frac{1}{z}\right)}{z n(z)},
$$

and for $\widehat{n}(z)$ in case (2.10) in a similar way or from [1, Lemma 3.3.6].

Conversely, starting from $\widetilde{n}(z)$ as in (2.8), the relation

$$
z\left(n(z)+\frac{s_{0}}{z}\right)=s_{0} \frac{\frac{\widetilde{n}(z)}{z}}{1+\frac{\widetilde{n}(z)}{z}}
$$

implies that from $\widetilde{n}(z)=\mathrm{o}(z)$ it follows that $n$ satisfies $\left(1_{0}\right)$. The corresponding proofs for (2.9) and (2.10) are similar.

\section{Self-adjoint operator representations}

A function $n \in \mathbf{N}_{0}$ admits a self-adjoint operator representation or realization with a self-adjoint relation $A$ in some Hilbert space $\mathcal{H}$ of the form

$$
n(z)=n\left(z_{0}\right)^{*}+\left(z-z_{0}^{*}\right)\left(\left(I+\left(z-z_{0}\right)(A-z)^{-1}\right) v, v\right)
$$

with $z_{0}$ an arbitrary non-real number $z_{0}$ and an element $v \in \mathcal{H}$, see [13, [10], and 9. If $v$ is chosen to be a generating element for $A$, which means that

$$
\mathcal{H}=\overline{\operatorname{span}}\left\{v,(A-z)^{-1} v \mid z \in \mathbb{C} \backslash \mathbb{R}\right\}
$$

and which is always possible, then the operator representation (3.2) is called minimal and then it is unique up to unitary equivalence. We have the following equivalences, see [15]:

$$
\begin{aligned}
A \text { is an operator } & \Longleftrightarrow n(z)=\mathrm{o}(z), \\
v \in \operatorname{dom} A & \Longleftrightarrow \lim _{y \rightarrow \infty} y \operatorname{Im} n(i y)<\infty ;
\end{aligned}
$$

for $n \in \mathbf{N}_{0}$ the latter limit always exists: it is either a non-negative number or $\infty$.

If the Nevanlinna function $n$ satisfies the assumption $\left(1_{0}\right)$ (or any of the assumptions $\left.\left(2_{0}\right),\left(3_{0}\right)\right)$ the representation (3.1) can be simplified to

$$
n(z)=\left((A-z)^{-1} u, u\right), \quad z \in \mathbb{C} \backslash \mathbb{R},
$$

where $A$ is a self-adjoint operator in some Hilbert space $\mathcal{H}, u \in \mathcal{H},(u, u)=s_{0}$. If $u$ is chosen to be a generating element for $A$, or equivalently,

$$
\mathcal{H}=\overline{\operatorname{span}}\left\{(A-z)^{-1} u \mid z \in \mathbb{C} \backslash \mathbb{R}\right\}
$$


which is always possible, then the operator representation (3.2) is also called min$\mathrm{imal}$ and then it is unique up to unitary equivalence. The representation (3.2) follows from (3.1) and the above mentioned equivalences by taking $u=c\left(A-z_{0}\right) v$ with some unimodular complex number $c$.

Here are three examples for a more concrete choice of the triplet $\mathcal{H}, A, u$ in (3.2) for the given function $n \in \mathbf{N}_{0}$ with integral representation (2.2).

(1) $\mathcal{H}=L^{2}(\sigma), A$ is the operator of multiplication with the independent variable, and $u(t) \equiv 1, t \in \mathbb{R}$.

(2) $\mathcal{H}$ is the completion of the linear span of the functions $\mathbf{r}_{z}, z \in \mathbb{C} \backslash \mathbb{R}$ :

$$
r_{z}(t):=\frac{1}{t-z}, \quad t \in \mathbb{R},
$$

with inner product defined by

$$
\left(r_{z}, r_{\zeta}\right)=\frac{n(z)-n(\zeta)^{*}}{z-\zeta^{*}}, \quad z, \zeta \in \mathbb{C} \backslash \mathbb{R}, z \neq \zeta^{*},
$$

$A$ is the operator of multiplication by $t$, and $u(t) \equiv 1, t \in \mathbb{R}$.

(3) $\mathcal{H}$ is the reproducing kernel Hilbert space $\mathcal{L}(n)$ with reproducing kernel

$$
L_{n}(z, w)=\frac{n(z)-n(w)^{*}}{z-w^{*}}, \quad z, w \in \mathbb{C} \backslash \mathbb{R}, z \neq w^{*},
$$

$A$ is the self-adjoint operator whose resolvent $(A-z)^{-1}$ is the differencequotient operator $R_{z}$ :

$$
\left(R_{z} f\right)(\zeta)=\frac{f(\zeta)-f(z)}{\zeta-z}, \quad f \in \mathcal{L}(n),
$$

and take $u=n$; this function belongs to the space $\mathcal{L}(n)$, since $n$ satisfies the condition $\left(1_{0}\right)$. Recall that the reproducing property of the kernel $L_{n}$ is reflected in the inner product of the space $\mathcal{L}(n)$ :

$$
\left\langle f, L_{n}(\cdot, z\rangle_{\mathcal{L}(n)}=f(z), \quad f \in \mathcal{L}(n), z \in \mathbb{C} \backslash \mathbb{R} .\right.
$$

That (3.2) holds follows from

$$
\left(R_{z} n\right)(\zeta)=L_{n}\left(\zeta, z^{*}\right)
$$

and the reproducing property of the kernel $L_{n}$ :

$$
\begin{aligned}
\left\langle(A-z)^{-1} u, u\right\rangle_{\mathcal{L}(n)} & =\left\langle R_{z} n, n\right\rangle_{\mathcal{L}(n)} \\
= & \left\langle n, L_{n}\left(\cdot, z^{*}\right)\right\rangle_{\mathcal{L}(n)}^{*}=n\left(z^{*}\right)^{*}=n(z) .
\end{aligned}
$$

The unitary equivalence of the representations in (1) and (2) follows easily from the relation

$$
\left(r_{z}, r_{\zeta}\right)=\int_{-\infty}^{\infty} \frac{d \sigma(t)}{(t-z)\left(t-\zeta^{*}\right)}, \quad z, \zeta \in \mathbb{C} \backslash \mathbb{R},
$$


and the fact that the functions $r_{z}, z \neq z^{*}$, form a total set in $L^{2}(\sigma)$. The unitary equivalence between the two representations of $n$ in (2) and (3) is given by the mapping $U$ :

$$
U\left(r_{z}\right)=L_{n}\left(\cdot, z^{*}\right)
$$

in particular, we have $U u=n$ where $u$ is the function $u(t) \equiv 1, t \in \mathbb{R}$. The space $L^{2}(\sigma)$ (or the equivalent space in $(2)$ ) we denote also by $\mathcal{H}(n)$. We mention that the definition of the spaces in (2) and (3) can also be used for generalized Nevanlinna functions, whereas in this case the space $L^{2}(\sigma)$ need not be defined. In Sections 4- 7 we will prove theorems using the representation of $n$ in (1), in Section 8 we reprove some of these results using the representation in the reproducing kernel Hilbert space $\mathcal{L}(n)$.

Since, according to Theorem 2.2, the functions $\widetilde{n}$ and $\widehat{n}$ in Definition 2.1 belong to the class $\mathbf{N}_{0}$ and are $\mathrm{o}(z)$ for $z=i y, y \rightarrow \infty$, they admit again an operator representation of the form (3.1), for example,

$$
\widehat{n}(z)=\widehat{n}\left(z_{0}\right)^{*}+\left(z-z_{0}^{*}\right)\left(\left(\widehat{A}-z_{0}\right)(\widehat{A}-z)^{-1} \widehat{v}, \widehat{v}\right)
$$

with a self-adjoint operator $\widehat{A}$ in some Hilbert space $\widehat{\mathcal{H}}, z_{0}$ an arbitrary non-real number, and an element $\widehat{v} \in \widehat{\mathcal{H}}$. Clearly, as the difference between the functions $\widetilde{n}$ and $\widehat{n}$ is just an additive real constant, the operator representation for $\widetilde{n}$ can be chosen the same, that is, in (3.3) $\widehat{n}$ can be replaced by $\widetilde{n}$.

Theorem 3.1. Let $n \in \mathbf{N}_{0}$ satisfying the condition (1 $\left.1_{0}\right)$ and with operator representation (3.2) be given, and let

$$
\widehat{n}(z)=\frac{-s_{0}}{n(z)}-z+\frac{s_{1}}{s_{0}}
$$

be the Schur transform of $n$ from (2.7). Then in the operator representation (3.3) of $\widehat{n}$ we can choose $\widehat{\mathcal{H}}=\{u\}^{\perp}, \widehat{A}$ in $\widehat{\mathcal{H}}$ as the compression of $A$ to $\widehat{\mathcal{H}}: \widehat{A}=\left.\widehat{P} A\right|_{\widehat{\mathcal{H}}}$, where $\widehat{P}$ is the orthogonal projection in $\mathcal{H}$ onto $\widehat{\mathcal{H}}$, and the element $\widehat{v}$ as

$$
\widehat{v}=\frac{\|u\|}{\left(\left(A-z_{0}\right)^{-1} u, u\right)} \widehat{P}\left(A-z_{0}\right)^{-1} u .
$$

If $\widehat{n}$ also satisfies the condition $\left(1_{0}\right) 1$, then

$$
\left.\widehat{n}(z)=\left((\widehat{A}-z)^{-1} \widehat{u}, \widehat{u}\right)\right), \quad \widehat{u}:=\frac{\widehat{P} A u}{\|u\|} .
$$

Remark 3.2. The resolvent of $\widehat{A}$ is given by

$$
(\widehat{A}-z)^{-1}=(A-z)^{-1}-\frac{\left((A-z)^{-1} \cdot u\right)}{\left((A-z)^{-1} u, u\right)}(A-z)^{-1} u,
$$

\footnotetext{
${ }^{1}$ This is the case when $n$ satisfies condition $\left(1_{1}\right)$ defined in Section 4 see Lemma 4.1
} 
and

$$
\widehat{v}=\frac{\|u\|\left(A-z_{0}\right)^{-1} u-\left(\left(A-z_{0}\right)^{-1} u, u\right) \frac{u}{\|u\|}}{\left|\left(\left(A-z_{0}\right)^{-1} u, u\right)\right|} .
$$

Note that $\left(\left(A-z_{0}\right)^{-1} u, u\right)=n\left(z_{0}\right) \neq 0$, otherwise $n(z) \equiv 0$.

Proof of Theorem 3.1. (1) Suppose that $n$ satisfies $\left(1_{0}\right)$. Then we have $\|u\|=\sqrt{s_{0}}$ and

$$
\widehat{n}(z)-\widehat{n}\left(z_{0}\right)^{*}=-\frac{\|u\|^{2}}{r(z)}-z+\frac{\|u\|^{2}}{r\left(z_{0}\right)^{*}}+z_{0}^{*},
$$

where we have put $r(z):=\left((A-z)^{-1} u, u\right)$. It remains to show that the expression on the right hand side of (3.4) equals

$\left.\left(z-z_{0}^{*}\right)\left(\left(\widehat{A}-z_{0}\right)(\widehat{A}-z)^{-1}\right) \widehat{v}, \widehat{v}\right)=\left(z-z_{0}^{*}\right)\|\widehat{v}\|^{2}+\left(z-z_{0}^{*}\right)\left(z-z_{0}\right)\left((\widehat{A}-z)^{-1} \widehat{v}, \widehat{v}\right)$.

This is a straightforward calculation, we only indicate some formulas:

$$
\begin{aligned}
\left(z-z_{0}\right)(\widehat{A}-z)^{-1} \widehat{v} & =\frac{\|u\|}{\left|r\left(z_{0}\right)\right|}\left(\frac{r\left(z_{0}\right)}{r(z)}(A-z)^{-1} u-\left(A-z_{0}\right)^{-1} u\right), \\
\|\widehat{v}\|^{2} & =\|u\|^{2} \frac{\left\|\left(A-z_{0}\right)^{-1} u\right\|^{2}}{\left|r\left(z_{0}\right)\right|^{2}}-1,
\end{aligned}
$$

and

$$
\begin{aligned}
& \left(z-z_{0}^{*}\right)\left(z-z_{0}\right)\left((\widehat{A}-z)^{-1} \widehat{v}, \widehat{v}\right) \\
& \quad=\|u\|^{2}\left(\frac{1}{r\left(z_{0}\right)^{*}}-\frac{1}{r(z)}-\frac{\left(z-z_{0}^{*}\right)\left\|\left(A-z_{0}\right)^{-1} u\right\|^{2}}{\left|r\left(z_{0}\right)\right|^{2}}\right) .
\end{aligned}
$$

(2) Now assume that $\widehat{n}$ satisfies $\left(1_{0}\right)$. Then $\widehat{v} \in \operatorname{dom} \widehat{A} \subset \operatorname{dom} A$ and the equality

$$
\widehat{v}=\frac{\|u\|}{r\left(z_{0}\right)}\left(A-z_{0}\right)^{-1} u-\frac{u}{\|u\|}
$$

shows that also $u \in \operatorname{dom} A$. If we take $\widehat{u}=-\left(\widehat{A}-z_{0}\right) \widehat{v}$, then (see after (3.2) $) \widehat{n}$ has the asserted representation. It remains to show that $\widehat{u}=\widehat{P} A u /\|u\|$. We have

$$
\widehat{u}=-\left(A-z_{0}\right) \widehat{v}=\frac{1}{\|u\|}\left(A-z_{0}\right) u-\frac{\|u\|}{r\left(z_{0}\right)} u .
$$

Taking the inner product of both sides with $u$ and using $(\widehat{u}, u)=0$, we see that

$$
\frac{\|u\|}{r\left(z_{0}\right)}=\frac{\left(\left(A-z_{0}\right) u, u\right)}{\|u\|^{3}}
$$

and hence

$$
\widehat{u}=\frac{1}{\|u\|}\left(A-z_{0}\right) u-\frac{\left(\left(A-z_{0}\right) u, u\right)}{\|u\|^{3}} u=\frac{1}{\|u\|}\left(A u-\frac{(A u, u)}{\|u\|^{2}} u\right)=\frac{1}{\|u\|} \widehat{P} A u .
$$




\section{Higher order asymptotics. Orthogonal polynomials}

1. For $n \in \mathbf{N}_{0}$ and some integer $p \geq 1$ we introduce the assumptions

$$
\begin{aligned}
& \left(1_{p}\right) n(z)=-\frac{s_{0}}{z}-\frac{s_{1}}{z^{2}}-\cdots-\frac{s_{2 p}}{z^{2 p+1}}+\mathrm{o}\left(\frac{1}{z^{2 p+1}}\right), \\
& \left(2_{p}\right) n(z)=-\frac{s_{0}}{z}-\frac{s_{1}}{z^{2}}-\cdots-\frac{s_{2 p}}{z^{2 p+1}}+\mathrm{O}\left(\frac{1}{z^{2 p+2}}\right), \\
& \left(3_{p}\right) n(z)=-\frac{s_{0}}{z}-\frac{s_{1}}{z^{2}}-\cdots-\frac{s_{2 p+1}}{z^{2 p+2}}+\mathrm{o}\left(\frac{1}{z^{2 p+2}}\right) .
\end{aligned}
$$

Again, $\left(3_{p}\right) \Longrightarrow\left(2_{p}\right) \Longrightarrow\left(1_{p}\right)$, and by [13, Satz 1.10] for the operator representation the assumption $\left(1_{p}\right)$ is equivalent to $u \in \operatorname{dom} A^{p}$. That is, for the above representation with the space $\mathcal{H}(n)$ the functions

$$
\mathbf{t}_{k}(t):=t^{k}, \quad k=0,1, \ldots, p,
$$

belong to $\mathcal{H}(n)$ and the first $p$ of these elements, $\mathbf{t}_{0}, \mathbf{t}_{1}, \ldots, \mathbf{t}_{p-1}$, belong to $\operatorname{dom} A$. Moreover, the formal relation

$$
(A-z)^{-1}=-\sum_{j=0}^{2 p} \frac{A^{j}}{z^{j+1}}+\frac{A^{2 p+1}}{z^{2 p+1}}(A-z)^{-1}
$$

implies easily

$$
\begin{aligned}
n(z) & =\left((A-z)^{-1} u, u\right) \\
& =-\sum_{j=0}^{p} \frac{\left(A^{j} u, u\right)}{z^{j+1}}-\sum_{j=p+1}^{2 p} \frac{\left(A^{n} u, A^{j-p} u\right)}{z^{j+1}}+\frac{1}{z^{2 p+1}}\left(A(A-z)^{-1} A^{p} u, A^{p} u\right) .
\end{aligned}
$$

It follows that

$$
s_{j}=\left\{\begin{array}{lll}
\left(A^{j} u, u\right) & \text { if } \quad j=0,1, \ldots, p, \\
\left(A^{n} u, A^{j-p} u\right) & \text { if } \quad j=p+1, p+2, \ldots, 2 p .
\end{array}\right.
$$

Therefore the above assumptions are equivalent to the following relations for the operator $A$ and the generating element $u, u \in \operatorname{dom} A^{p}$ :

$$
\begin{aligned}
& \left(1_{p}\right) \Longleftrightarrow u \in \operatorname{dom} A^{p}, \\
& \left(2_{p}\right) \Longleftrightarrow u \in \operatorname{dom} A^{p}, z\left(A(A-z)^{-1} A^{p} u, A^{p} u\right)=\mathrm{O}(1), \\
& \left(3_{p}\right) \Longleftrightarrow u \in \operatorname{dom} A^{p}, z\left(A(A-z)^{-1} A^{p} u, A^{p} u\right)+\alpha=\mathrm{o}(1) \text { with } \alpha \in \mathbb{R} ;
\end{aligned}
$$

in fact, in the last equivalence we have $\alpha=s_{2 p+1}$. 
Now we consider a function $n \in \mathbf{N}_{0}$ with the property $\left(1_{p}\right)$ for some $p>1$. For $0 \leq k \leq p$, by $S_{k}$ we denote the $(k+1) \times(k+1)$ Hankel matrix

$$
S_{k}:=\left(\begin{array}{cccc}
s_{0} & s_{1} & \cdots & s_{k} \\
s_{1} & s_{2} & \cdots & s_{k+1} \\
\vdots & \vdots & & \vdots \\
s_{k} & s_{k+1} & \cdots & s_{2 k}
\end{array}\right)
$$

it is the Gram matrix associated with the $k+1$ functions $\mathbf{t}_{0}, \mathbf{t}_{1}, \ldots, \mathbf{t}_{k}$, and we introduce the Gram determinants

$$
D_{k}:=\operatorname{det} S_{k}=\left|\begin{array}{cccc}
s_{0} & s_{1} & \ldots & s_{k} \\
s_{1} & s_{2} & \cdots & s_{k+1} \\
\vdots & \vdots & & \vdots \\
s_{k} & s_{k+1} & \cdots & s_{2 k}
\end{array}\right|, \quad k=0,1, \ldots p .
$$

Further, for $k=1, \ldots, p, \mathcal{H}_{k}$ denotes the $k$-dimensional subspace

$$
\mathcal{H}_{k}:=\operatorname{span}\left\{\mathbf{t}_{0}, \mathbf{t}_{1}, \ldots, \mathbf{t}_{k-1}\right\}
$$

of $\mathcal{H}(n)$. Evidently, the subspace $\mathcal{H}_{k}$ is non-degenerated if and only if $D_{k-1} \neq 0$.

In the rest of this section we suppose that $D_{p-1} \neq 0$, that is, the subspace $\mathcal{H}_{p}$ is non-degenerated. If $D_{p}=0$, then the function $n$ with the given asymptotics $\left(j_{p}\right)$ is uniquely determined and rational of Mac Millan degree $p$, in fact, see 11, pp. 22,23]

$$
n(z)=-\frac{d_{p}(z)}{e_{p}(z)}
$$

where the polynomials $e_{p}$ of degree $p$ and $d_{p}$ of degree $p-1$ are defined below. To exclude this (simple) case we often suppose that even $D_{p} \neq 0$; clearly, this implies $D_{p-1} \neq 0$.

As a basis in $\mathcal{H}_{p}$ we choose a system of elements $e_{k} \in \mathcal{H}(n)=L^{2}(\sigma), k=$ $0,1, \ldots, p-1$, which is obtained from the system $\mathbf{t}_{0}, \mathbf{t}_{1}, \ldots, \mathbf{t}_{p}$ by the GramSchmidt orthonormalization procedure. This so-called system of orthogonal polynomials of the first kind, associated with the function $n$ is defined by the following properties, $j, k=0,1, \ldots, p-1$ :

1. $e_{0}(z) \equiv 1 / \sqrt{s_{0}}$,

2. $e_{k}(z)$ is a real polynomial of degree $k$ with positive leading coefficient,

3. $\left(e_{j}, e_{k}\right)=\delta_{j k}$.

Then, see [1, (1.4)],

$$
e_{k}(z)=\frac{1}{\sqrt{D_{k-1} D_{k}}}\left|\begin{array}{cccc}
s_{0} & s_{1} & \cdots & s_{k} \\
s_{1} & s_{2} & \cdots & s_{k+1} \\
\vdots & \vdots & \cdots & \vdots \\
s_{k-1} & s_{k} & \cdots & s_{2 k-1} \\
1 & z & \cdots & z^{k}
\end{array}\right|, \quad k=1,2, \ldots, p-1,
$$


and by this formula with $k=p$ also a polynomial $e_{p}$ can be defined. Evidently, $e_{p} \in \mathcal{H}_{p}^{[\perp]}$, and

$$
\operatorname{span}\left\{\mathbf{t}_{0}, \mathbf{t}_{1}, \ldots, \mathbf{t}_{k}\right\}=\operatorname{span}\left\{e_{0}, e_{1}, \ldots, e_{k}\right\}, \quad k=0,1, \ldots, p .
$$

The orthogonal polynomials $e_{j}, j=0,1, \ldots, p$, satisfy the difference equations

$$
b_{k-1} e_{k-1}(z)+a_{k} e_{k}(z)+b_{k} e_{k+1}(z)=z e_{k}(z), \quad k=0,1, \ldots, p-1,
$$

with real numbers $a_{k}, k=0,1, \ldots, p-1, b_{-1}=0$, and positive numbers $b_{k}, k=$ $1, \ldots, p-1$, and the 'initial condition' $e_{0}(z)=1 / \sqrt{s_{0}}$. Explicit formulas for $a_{k}, b_{k}$ can be given, see [1; we note that

$$
a_{0}=\frac{s_{1}}{s_{0}}, \quad b_{0}=\frac{\sqrt{s_{2} s_{0}-s_{1}^{2}}}{s_{0}} .
$$

The relation (4.5) implies that with respect to the basis $e_{0}, e_{1}, \ldots, e_{p-1}$ of the space $\mathcal{H}_{p}$ the compression $A_{p}$ of the operator $A$ to $\mathcal{H}_{p}$ is given by the Jacobi matrix

$$
\mathcal{A}_{p}:=\left(\begin{array}{cccccc}
a_{0} & b_{0} & 0 & \cdots & 0 & 0 \\
b_{0} & a_{1} & b_{1} & \cdots & 0 & 0 \\
\vdots & \vdots & \vdots & \vdots & \vdots & \vdots \\
0 & 0 & 0 & \cdots & a_{p-2} & b_{p-2} \\
0 & 0 & 0 & \cdots & b_{p-2} & a_{p-1}
\end{array}\right),
$$

and that

$$
A e_{p-1}=b_{p-2} e_{p-2}+a_{p-1} e_{p-1}+b_{p-1} e_{p} .
$$

The latter relation means for the orthogonal polynomials

$$
b_{p-2} e_{p-2}(z)+a_{p-1} e_{p-1}(z)+b_{p-1} e_{p}(z)=z e_{p-1}(z),
$$

therefore, the eigenvalues of $\mathcal{A}_{p}$ are the zeros of the polynomial $e_{p}$. For later use we write the last $p-1$ difference equations (4.5) explicitly in the form

$$
\left\{\begin{aligned}
b_{0} e_{0}+a_{1} e_{1}+b_{1} e_{2} & =z e_{1} \\
b_{1} e_{1}+a_{2} e_{2}+b_{2} e_{3} & =z e_{2} \\
b_{2} e_{2}+a_{3} e_{3}+b_{3} e_{4} & =z e_{3} \\
& \vdots \\
b_{p-2} e_{p-2}+a_{p-1} e_{p-1}+b_{p-1} e_{p} & =z e_{p-1}
\end{aligned}\right.
$$

this system of homogeneous equations for $e_{0}, e_{1}, \ldots, e_{p}$ determines the orthogonal polynomials uniquely if we add the initial conditions

$$
e_{0}(z)=\frac{1}{\sqrt{s_{0}}}, \quad e_{1}(z)=\frac{z-a_{0}}{b_{0} \sqrt{s_{0}}} ;
$$

the second condition is just the first equation in 4.5. . 
The polynomials of the second kind, associated with the function $n \in \mathbf{N}_{0}$, are the functions $d_{k}, k=0,1, \ldots, p$, defined as follows:

$$
d_{k}(z)=\sqrt{s_{0}}\left(\frac{e_{k}(z)-e_{k}(\cdot)}{z-\cdot}, e_{0}\right)=\left(\frac{e_{k}(z)-e_{k}(\cdot)}{z-\cdot}, u\right), \quad k=0,1, \ldots, p .
$$

Hence $d_{0}(z)=0$ and $d_{k}$ is a polynomial of degree $k-1, k \geq 1$. The definition of $d_{k}$ and the relation (4.5) imply that

$$
b_{k-1} d_{k-1}(z)+a_{k} d_{k}(z)+b_{k} d_{k+1}(z)=z d_{k}(z), \quad k=1, \ldots, p-1 .
$$

Therefore the polynomials $e_{k}$ and $d_{k}$ satisfy for $k=1,2, \ldots, p-1$ the same difference equations but with different initial conditions:

$$
d_{0}(z)=0, \quad d_{1}(z)=\frac{\sqrt{s_{0}}}{b_{0}} .
$$

For later use we write the difference equations (4.11) in the form

$$
\begin{cases}a_{1} d_{1}+b_{1} d_{2} & =z d_{1} \\ b_{1} d_{1}+a_{2} d_{2}+b_{2} d_{3} & =z d_{2} \\ b_{2} d_{2}+a_{3} d_{3}+b_{3} d_{4} & =z d_{3} \\ & \vdots \\ b_{p-2} d_{p-2}+a_{p-1} d_{p-1}+b_{p-1} d_{p} & =z d_{p-1} .\end{cases}
$$

For any two solutions $u_{0}, \ldots, u_{p}$ and $v_{0}, \ldots, v_{p}$ of the difference equations (4.5) with $b_{-1}=0$ :

$$
\begin{aligned}
& z u_{k}(z)=b_{k-1} u_{k-1}(z)+a_{k} u_{k}(z)+b_{k} u_{k+1}(z), \quad k=0,1, \ldots, p-1, \\
& \zeta v_{k}(\zeta)=b_{k-1} v_{k-1}(\zeta)+a_{k} v_{k}(\zeta)+b_{k} v_{k+1}(\zeta),
\end{aligned}
$$

the Christoffel-Darboux formulas hold:

$$
\begin{aligned}
\sum_{k=m}^{p-1}(z-\zeta) u_{k}(z) v_{k}(\zeta)= & b_{p-1}\left(u_{p}(z) v_{p-1}(\zeta)-u_{p-1}(z) v_{p}(\zeta)\right) \\
& -b_{m-1}\left(u_{m}(z) v_{m-1}(\zeta)-u_{m-1}(z) v_{m}(\zeta)\right) ;
\end{aligned}
$$

in particular,

$$
d_{p}(z) e_{p-1}(z)-e_{p}(z) d_{p-1}(z)=\frac{1}{b_{p-1}} .
$$

2. In this subsection we assume that $n \in \mathbf{N}_{0}$ satisfies the assumption $\left(1_{p}\right)$ for some $p \geq 1$, and we consider its Schur transform $\widehat{n}$ from (2.7). For the following lemma see [8, Lemma 2.1], we sketch the proof.

Lemma 4.1. Suppose that $n$ satisfies $\left(1_{p}\right)$ for some $p \geq 1$ :

$$
n(z)=-\frac{s_{0}}{z}-\frac{s_{1}}{z^{2}}-\cdots-\frac{s_{2 p}}{z^{2 p+1}}+\mathrm{o}\left(\frac{1}{z^{2 p+1}}\right),
$$

then its Schur transform $\widehat{n}$ satisfies $\left(1_{p-1}\right)$ :

$$
\widehat{n}(z)=-\frac{\widehat{s}_{0}}{z}-\frac{\widehat{s}_{1}}{z^{2}}-\cdots-\frac{\widehat{s}_{2 p-2}}{z^{2 p-1}}+\mathrm{o}\left(\frac{1}{z^{2 p-1}}\right),
$$


with for $j=0,1, \ldots, 2 p-2$

$$
\widehat{s}_{j}=\frac{(-1)^{j+1}}{s_{0}^{j+2}}\left|\begin{array}{cccccc}
s_{1} & s_{0} & 0 & \cdots & 0 & 0 \\
s_{2} & s_{1} & s_{0} & \cdots & 0 & 0 \\
\vdots & \vdots & \ddots & \ddots & \vdots & \vdots \\
s_{j+1} & s_{j} & s_{j-1} & \cdots & s_{1} & s_{0} \\
s_{j+2} & s_{j+1} & s_{j} & \cdots & s_{2} & s_{1}
\end{array}\right|,
$$

Proof. Write

$$
\widehat{n}(z)=z\left(1+\frac{s_{1}}{s_{0} z}+\frac{s_{2}}{s_{0} z^{2}}+\cdots+\frac{s_{2 p}}{s_{0} z^{2 p}}+\mathrm{o}\left(\frac{1}{z^{2 p}}\right)\right)^{-1}-z+\frac{s_{1}}{s_{0}} .
$$

If we set

$$
q(z)=\frac{s_{1}}{s_{0} z}+\frac{s_{2}}{s_{0} z^{2}}+\cdots+\frac{s_{2 p}}{s_{0} z^{2 p}}+\mathrm{o}\left(\frac{1}{z^{2 p}}\right)
$$

then

$$
\begin{aligned}
\frac{1}{1+q(z)}= & 1-q(z)+\cdots-q(z)^{2 p-1}+\frac{q(z)^{2 p}}{1+q(z)} \\
& =1-q(z)+\cdots-q(z)^{2 p-1}+o\left(\frac{1}{z^{2 p-1}}\right)
\end{aligned}
$$

and

$$
\begin{aligned}
\widehat{n}(z) & =\frac{z}{1+q(z)}-\left(z-\frac{s_{1}}{s_{0}}\right) \frac{1+q(z)}{1+q(z)} \\
& =\left(-z q(z)+\frac{s_{1}}{s_{0}}(1+q(z))\right) \frac{1}{1+q(z)} \\
& =\left(-z\left(\frac{s_{2}}{s_{0} z^{2}}+\cdots+\frac{s_{2 p}}{s_{0} z^{2 p}}+\mathrm{o}\left(\frac{1}{z^{2 p}}\right)\right)+\frac{s_{1}}{s_{0}} q(z)\right) \frac{1}{1+q(z)} \\
& =\left(-\frac{s_{2}}{s_{0} z}-\cdots-\frac{s_{2 p}}{s_{0} z^{2 p-1}}+\mathrm{o}\left(\frac{1}{z^{2 p-1}}\right)+\frac{s_{1}}{s_{0}} q(z)\right) \frac{1}{1+q(z)},
\end{aligned}
$$

which is of the needed form. Formula (4.16) for the coefficients $\widehat{s}_{j}$ can be obtained by equating powers of $z$ from both sides of the equality

$$
n(z)\left(z-\frac{s_{1}}{s_{0}}+\widehat{n}(z)\right)=-s_{0} .
$$

Now we can formulate the main result of this subsection.

Theorem 4.2. Let $n \in \mathbf{N}_{0}$ satisfy condition $\left(1_{p}\right)$ for some $p \geq 2$. If $e_{k}$ and $d_{k}, k=0,1, \ldots, p$, denote the polynomials of first and second kind associated 
with the function $n$, and $\widehat{e}_{k}$ and $\widehat{d}_{k}, k=0,1, \ldots, p-1$, denote the polynomials of first and second kind associated with the Schur transform $\widehat{n}$ of $n$, then for $k=0,1, \ldots, p-1$ the following relations hold:

$$
\begin{gathered}
\widehat{e}_{k}(z)=\frac{1}{\sqrt{s_{0}}} d_{k+1}(z), \\
\widehat{d}_{k}(z)=-\sqrt{s_{0}} e_{k+1}(z)+\frac{1}{\sqrt{s_{0}}}\left(z-\frac{s_{1}}{s_{0}}\right) d_{k+1}(z) \\
=b_{0}\left(\frac{e_{k+1}(z)-e_{k+1}(\cdot)}{z-\cdot}, e_{1}\right)=\frac{1}{\sqrt{s_{0}}}\left(\frac{e_{k+1}(z)-e_{k+1}(\cdot)}{z-\cdot}, \cdot-a_{0}\right) .
\end{gathered}
$$

Remark 4.3. (i) Here we write $\widehat{e}$ and $\widehat{d}$ for the polynomials of first and second kind associated with the Schur transform $\widehat{n}$ of $n$, but the reader is reminded that these functions are not the Schur transforms of the polynomials $e$ and $d$.

(ii) If $\check{e}_{k}$ and $\check{d}_{k}$ stand for the polynomials of first and second kind associated with the Nevanlinna function $-1 / n$, then for $k=0,1, \ldots, p-1$

$$
\check{e}_{k}(z)=d_{k+1}(z), \quad \check{d}_{k}(z)=-e_{k+1}(z)+\frac{1}{s_{0}}\left(z-\frac{s_{1}}{s_{0}}\right) d_{k+1}(z) .
$$

The first equality readily follows from the fact that the spectral functions of $\widehat{n}$ and $-1 / n$ only differ by a factor $s_{0}$. The second equality can be obtained by tracing the proof below; the only difference lies in (4.19): with evident notation, it should be replaced by

$$
\check{s}_{0}=\frac{s_{0} s_{2}-s_{1}^{2}}{s_{0}^{3}} .
$$

Proof of Theorem 4.2. For the function $\widehat{n}$, again with evident notation, we have

$$
\widehat{s}_{0}=\frac{s_{0} s_{2}-s_{1}^{2}}{s_{0}^{2}}
$$

and, as a consequence of Theorem 3.1

$$
\widehat{\mathcal{A}}_{p-1}=\left(\begin{array}{cccccc}
\widehat{a}_{0} & \widehat{b}_{0} & 0 & \cdots & 0 & 0 \\
\widehat{b}_{0} & \widehat{a}_{1} & \widehat{b}_{1} & \cdots & 0 & 0 \\
\vdots & \vdots & \vdots & \vdots & \vdots & \vdots \\
0 & 0 & 0 & \cdots & \widehat{a}_{p-3} & \widehat{b}_{p-3} \\
0 & 0 & 0 & \cdots & \widehat{b}_{p-3} & \widehat{a}_{p-2}
\end{array}\right)=\left(\begin{array}{cccccc}
a_{1} & b_{1} & 0 & \cdots & 0 & 0 \\
b_{1} & a_{2} & b_{2} & \cdots & 0 & 0 \\
\vdots & \vdots & \vdots & \vdots & \vdots & \vdots \\
0 & 0 & 0 & \cdots & a_{p-2} & b_{p-2} \\
0 & 0 & 0 & \cdots & b_{p-2} & a_{p-1}
\end{array}\right) .
$$

For the $\widehat{e}_{j}, j=0, \ldots, p-1$, we find

$$
\widehat{e}_{0}(z)=\frac{1}{\sqrt{\widehat{s}_{0}}}=\frac{s_{0}}{\sqrt{s_{0} s_{2}-s_{1}^{2}}}=\frac{1}{b_{0}}=\frac{1}{\sqrt{s_{0}}} d_{1}(z)
$$


and $\widehat{e}_{1}, \widehat{e}_{2}, \ldots, \widehat{e}_{p-1}$ follow from the equations (see (4.5)):

$$
\begin{cases}a_{1} \widehat{e}_{0}+b_{1} \widehat{e}_{1} & =z \widehat{e}_{0} \\ b_{1} \widehat{e}_{0}+a_{2} \widehat{e}_{1}+b_{2} \widehat{e}_{2} & =z \widehat{e}_{1} \\ b_{2} \widehat{e}_{1}+a_{3} \widehat{e}_{2}+b_{3} \widehat{e}_{3} & =z \widehat{e}_{2} \\ & \vdots \\ b_{p-2} \widehat{e}_{p-3}+a_{p-1} \widehat{e}_{p-2}+b_{p-1} \widehat{e}_{p-1} & =z \widehat{e}_{p-2}\end{cases}
$$

Since these equation coincide with (4.13) we obtain

$$
\widehat{e}_{j}=c d_{j+1}, \quad j=0,1, \ldots, p-1 .
$$

The constant $c$ can be determined from the initial condition $\widehat{e}_{0}=c d_{1}$, which gives $c=1 / \sqrt{s_{0}}$. Therefore

$$
\widehat{e}_{j}(z)=\frac{1}{\sqrt{s_{0}}} d_{j+1}(z), \quad j=0,1, \ldots, p-1,
$$

and (4.17) is proved.

For the polynomials of second kind $\widehat{d}_{j}$ we obtain in a similar way

$$
\begin{gathered}
\widehat{d}_{0}(z)=0, \quad \widehat{d}_{1}(z)=\frac{\sqrt{\widehat{s}_{0}}}{\widehat{b}_{0}} \\
\begin{cases}\widehat{a}_{1} \widehat{d}_{1}+\widehat{b}_{1} \widehat{d}_{2} \\
\widehat{b}_{1} \widehat{d}_{1}+\widehat{a}_{2} \widehat{d}_{2}+\widehat{b}_{2} \widehat{d}_{3} & =z \widehat{d}_{1} \\
\widehat{b}_{2} \widehat{d}_{2}+\widehat{a}_{3} \widehat{d}_{3}+\widehat{b}_{3} \widehat{d}_{4} & =z \widehat{d}_{3} \\
\widehat{b}_{p-3} \widehat{d}_{p-3}+\widehat{a}_{p-2} \widehat{d}_{p-2}+\widehat{b}_{p-2} \widehat{d}_{p-1} & =z \widehat{d}_{p-2}\end{cases}
\end{gathered}
$$

(one equation less than in (4.13)). These equations can be written as

$$
\left\{\begin{aligned}
a_{2} \widehat{d}_{1}+b_{2} \widehat{d}_{2} & =z \widehat{d}_{1} \\
b_{2} \widehat{d}_{1}+a_{3} \widehat{d}_{2}+b_{3} \widehat{d}_{3} & =z \widehat{d}_{2} \\
b_{3} \widehat{d}_{2}+a_{4} \widehat{d}_{3}+b_{4} \widehat{d}_{4} & =z \widehat{d}_{3} \\
b_{p-2} \widehat{d}_{p-3}+a_{p-1} \widehat{d}_{p-2}+b_{p-1} \widehat{d}_{p-1} & =z \widehat{d}_{p-2} .
\end{aligned}\right.
$$

The last $p-3$ equations of this system coincide with the last $p-3$ equations of (4.8) and (4.13). Therefore a solution vector $\left(\widehat{d}_{j}\right)_{1}^{p-1}$ of the last $p-3$ equations of (4.21) can be obtained as a linear combination of the solution vectors $\left(e_{j}\right)_{2}^{p}$ and $\left(d_{j}\right)_{2}^{p}$ of the last $p-3$ equations in (4.8) and (4.13):

$$
\widehat{d}_{j}=\gamma e_{j+1}+\delta d_{j+1}, \quad j=1,2, \ldots, p-1 .
$$


Now $\gamma, \delta$ have to be found such that these relations hold also for $j=0$ with $\widehat{d}_{0}(z)=0$, and for $j=1$ with $\widehat{d}_{1}(z)=\frac{\sqrt{\widehat{s}_{0}}}{\widehat{b}_{0}}$. Since $\widehat{d}_{0}(z)=0$ it follows that

$$
0=\gamma e_{1}(z)+\delta d_{1}(z)=\gamma \frac{z-a_{0}}{b_{0}} \frac{1}{\sqrt{s_{0}}}+\delta \frac{\sqrt{s_{0}}}{b_{0}}=\gamma \frac{z-\frac{s_{1}}{s_{0}}}{b_{0}} \frac{1}{\sqrt{s_{0}}}+\delta \frac{\sqrt{s_{0}}}{b_{0}},
$$

which is satisfied for

$$
\gamma=-\varepsilon, \quad \delta=\varepsilon\left(\frac{z}{s_{0}}-\frac{s_{1}}{s_{0}^{2}}\right) .
$$

The relation $\widehat{d}_{1}(z)=\frac{\sqrt{\widehat{s}_{0}}}{\widehat{b}_{0}}$ implies

$$
\begin{aligned}
\widehat{d}_{1}(z) & =\varepsilon\left(-e_{2}(z)+\left(\frac{z}{s_{0}}-\frac{a_{0}}{s_{0}}\right) d_{2}(z)\right) \\
& =\varepsilon\left(-\frac{\left(z-a_{1}\right) e_{1}-b_{0} e_{0}}{b_{1}}+\frac{z-a_{0}}{s_{0}} \frac{\left(z-a_{1}\right) d_{1}}{b_{1}}\right) \\
& =\varepsilon\left(-\frac{\left(z-a_{1}\right) \frac{z-a_{0}}{b_{0}} \frac{1}{\sqrt{s_{0}}}-b_{0} \frac{1}{\sqrt{s_{0}}}}{b_{1}}+\frac{z-a_{0}}{s_{0}} \frac{\left(z-a_{1}\right) \frac{\sqrt{s_{0}}}{b_{0}}}{b_{1}}\right) \\
& =\varepsilon \frac{b_{0}}{b_{1} \sqrt{s_{0}}}=\frac{\sqrt{\widehat{s}_{0}}}{\widehat{b}_{0}}=\frac{b_{0}}{\widehat{b}_{0}},
\end{aligned}
$$

hence

$$
\varepsilon=\frac{b_{1}}{\widehat{b}_{0}} \sqrt{s_{0}} .
$$

According to (4.20) we find $\varepsilon=\sqrt{s_{0}}$. This proves the first equality in (4.18). The remaining equalities follow from (4.10) and the second equality in (4.9).

3. In this subsection we give a second proof of Theorem 4.2 using asymptotic expansions, see [1, (1.34b)]: Assume that $n \in \mathbf{N}_{0}$ satisfies $\left(1_{p}\right)$ for some $p \geq 2$, that is,

$$
n(z)=-\frac{s_{0}}{z}-\frac{s_{1}}{z^{2}}-\cdots-\frac{s_{2 p}}{z^{2 p+1}}+\mathrm{o}\left(\frac{1}{z^{2 p+1}}\right),
$$

then

$$
-\frac{d_{p}(z)}{e_{p}(z)}=-\frac{s_{0}}{z}-\frac{s_{1}}{z^{2}}-\cdots-\frac{s_{2 p-1}}{z^{2 p}}+\mathrm{O}\left(\frac{1}{z^{2 p+1}}\right) .
$$

According to [1, the second to last formula on p. 22] the function $-d_{p} / e_{p}$ is a Nevanlinna function and by [13, Bemerkung 1.11] there is a real number $t_{2 p}$ such that $-d_{p} / e_{p}$ has the asymptotic expansion

$$
-\frac{d_{p}(z)}{e_{p}(z)}=-\frac{s_{0}}{z}-\frac{s_{1}}{z^{2}}-\cdots-\frac{s_{2 p-1}}{z^{2 p}}-\frac{t_{2 p}}{z^{2 p+1}}+\mathrm{o}\left(\frac{1}{z^{2 p+1}}\right) .
$$


By Lemma 4.1,

$$
\widehat{n}(z)=-\frac{\widehat{s}_{0}}{z}-\frac{\widehat{s}_{1}}{z^{2}}-\cdots-\frac{\widehat{s}_{2 p-2}}{z^{2 p-1}}+\mathrm{o}\left(\frac{1}{z^{2 p-1}}\right),
$$

and hence by (4.22)

$$
-\frac{\widehat{d}_{p-1}(z)}{\widehat{e}_{p-1}(z)}=-\frac{\widehat{s}_{0}}{z}-\frac{\widehat{s}_{1}}{z^{2}}-\cdots-\frac{\widehat{s}_{2 p-3}}{z^{2 p-2}}+\mathrm{O}\left(\frac{1}{z^{2 p-1}}\right) .
$$

By Lemma 4.1, the Schur transform of the function $-d_{p} / e_{p}$ in (4.23) has the asymptotic expansion

$$
\begin{aligned}
& \widehat{\left(-\frac{d_{p}}{e_{p}}\right)}(z)=\frac{s_{0} e_{p}(z)}{d_{p}(z)}-z+\frac{s_{1}}{s_{0}}=: \frac{r(z)}{d_{p}(z)} \\
& =-\frac{\widehat{s}_{0}}{z}-\frac{\widehat{s}_{1}}{z^{2}}-\cdots-\frac{\widehat{s}_{2 p-3}}{z^{2 p-2}}-\frac{\widehat{t}_{2 p-2}}{z^{2 p-1}}+\mathrm{o}\left(\frac{1}{z^{2 p-1}}\right), \\
& =-\frac{\widehat{s}_{0}}{z}-\frac{\widehat{s}_{1}}{z^{2}}-\cdots-\frac{\widehat{s}_{2 p-3}}{z^{2 p-2}}+\mathrm{O}\left(\frac{1}{z^{2 p-1}}\right)
\end{aligned}
$$

where only the number $\widehat{t}_{2 p-2}$ depends on $t_{2 p}$ according to formula (4.16). Here the polynomial $r$, defined via the second equality sign, is given by

$$
r(z)=s_{0} e_{p}(z)-\left(z-\frac{s_{1}}{s_{0}}\right) d_{p}(z)
$$

and its degree is $\leq p$. Comparing (4.24) with (4.25), we find that

$$
\frac{r(z)}{d_{p}(z)}-\frac{\widehat{d}_{p-1}(z)}{\widehat{e}_{p-1}(z)}=\mathrm{O}\left(\frac{1}{z^{2 p-1}}\right) \text {. }
$$

The degree of the product $d_{p} \widehat{e}_{p-1}$ equals $2 p-2$ and hence

$$
\frac{r(z)}{d_{p}(z)}=\frac{\widehat{d}_{p-1}(z)}{\widehat{e}_{p-1}(z)}
$$

which readily implies that for some number $k \neq 0$

$$
\widehat{e}_{p-1}(z)=k d_{p}(z), \quad \widehat{d}_{p-1}(z)=k\left(s_{0} e_{p}(z)-\left(z-\frac{s_{1}}{s_{0}}\right) d_{p}(z)\right) .
$$

We claim $k=1 / \sqrt{s_{0}}$. With the proof of the claim the proof of the theorem is complete.

To prove the claim we note that the leading coefficient of the polynomial $e_{k}$ is equal to $\sqrt{D_{k-1} / D_{k}}$ and that, by (4.5),

$$
\sqrt{\frac{D_{k}}{D_{k+1}}}=\frac{1}{b_{k}} \sqrt{\frac{D_{k-1}}{D_{k}}} .
$$

Hence

$$
\sqrt{\frac{D_{p-1}}{D_{p}}}=\frac{1}{b_{p-1}} \cdots \frac{1}{b_{1}} \sqrt{\frac{D_{0}}{D_{1}}}=\frac{1}{b_{p-1}} \cdots \frac{1}{b_{1}} \frac{1}{b_{0}} \frac{1}{\sqrt{s_{0}}}
$$


and, similarly, because of (4.20) and (4.19),

$$
\sqrt{\frac{\widehat{D}_{p-2}}{\widehat{D}_{p-1}}}=\frac{1}{\widehat{b}_{p-2}} \cdots \frac{1}{\widehat{b}_{0}} \frac{1}{\sqrt{\widehat{s}_{0}}}=\frac{1}{b_{p-1}} \cdots \frac{1}{b_{1}} \frac{1}{b_{0}} .
$$

From (4.26) we obtain

$$
\frac{1}{k} \sqrt{\frac{\widehat{D}_{p-2}}{\widehat{D}_{p-1}}}=\lim _{z \rightarrow \infty} \frac{d_{p}(z)}{z^{p-1}}=s_{0} \lim _{z \rightarrow \infty} \frac{e_{p}(z)}{z^{p}}=s_{0} \sqrt{\frac{D_{p-1}}{D_{p}}},
$$

that is,

$$
\sqrt{s_{0}} \frac{1}{b_{p-1}} \cdots \frac{1}{b_{1}} \frac{1}{b_{0}}=\frac{1}{k} \frac{1}{b_{p-1}} \cdots \frac{1}{b_{1}} \frac{1}{b_{0}} .
$$

Therefore, $k=1 / \sqrt{s_{0}}$ and the claim holds.

\section{Reduction via a $p$-dimensional subspace}

Let again $n \in \mathbf{N}_{0}$ with the property $\left(1_{p}\right)$ be given. We decompose the space $\mathcal{H}(n)$ with $\mathcal{H}_{p}=\operatorname{span}\left\{\mathbf{t}_{0}, \mathbf{t}_{1}, \ldots, \mathbf{t}_{p-1}\right\}$ as follows:

$$
\mathcal{H}(n)=\mathcal{H}_{p} \oplus \mathcal{H}_{p}^{\prime} .
$$

Then, evidently, $e_{p} \in \mathcal{H}_{p}^{\prime}$. The corresponding matrix representation of the operator $A$ is

$$
A=\left(\begin{array}{cc}
A_{0} & \widetilde{B} \\
B & D
\end{array}\right)
$$

with $A_{0}$ given by the Jacobi matrix $\mathcal{A}_{0}$ from (4.7),

$$
B=b_{p-1}\left(\cdot, e_{p-1}\right) e_{p}, \quad \widetilde{B}=b_{p-1}\left(\cdot, e_{p}\right) e_{p-1} .
$$

The operator $A_{0}$ is bounded and self-adjoint in $\mathcal{H}_{p}$, and $D$ is self-adjoint (possibly unbounded) in $\mathcal{H}_{p}^{\prime}$. In the following theorem we express the function $n$ by means of the entries of the matrix in (5.2). We set

$$
\begin{gathered}
a_{00}(z):=\left(\left(A_{0}-z\right)^{-1} u, u\right), \quad a_{11}(z):=\left(\left(A_{0}-z\right)^{-1} e_{p-1}, e_{p-1}\right), \\
a(z):=\left(\left(A_{0}-z\right)^{-1} u, e_{p-1}\right)=\left(\left(A_{0}-z\right)^{-1} e_{p-1}, u\right),
\end{gathered}
$$

and

$$
\check{r}(z):=\left|\begin{array}{cc}
a_{00}(z) & a(z) \\
a(z) & a_{11}(z)
\end{array}\right| .
$$

The last equality in (5.3) follows from $a\left(z^{*}\right)^{*}=a(z)$, in fact, by Cramer's rule, $a(z)=\sqrt{s_{0}} /\left(a_{0}-z\right)$ if $p=1$ and $a(z)=(-1)^{p-1} \sqrt{s_{0}} b_{0} \ldots b_{p-2} / \operatorname{det}\left(\mathcal{A}_{0}-z\right)$ if $p \geq 2$. 
Theorem 5.1. Suppose that the function $n \in \mathbf{N}_{0}$ satisfies for some $p \geq 1$ one of the assumptions $\left(j_{p}\right), j=1,2,3$, and that $D_{p} \neq 0$, see (4.3). Then

$$
n(z)=\frac{\check{r}(z) n_{p}(z)-a_{00}(z)}{a_{11}(z) n_{p}(z)-1},
$$

where $n_{p}(z):=\left((D-z)^{-1} u_{p}, u_{p}\right)$ with $u_{p}:=b_{p-1} e_{p}$. The function $n_{p}$ belongs to $\mathbf{N}_{0}$ and satisfies the assumption $\left(j_{0}\right)$. Moreover, for $k \geq 1$ we have

$$
u \in \operatorname{dom} A^{p+k} \Longleftrightarrow u_{p} \in \operatorname{dom} D^{k}
$$

and $n$ satisfies the assumption $\left(j_{p+k}\right)$ if and only if $n_{p}$ satisfies the assumption $\left(j_{k}\right)$.

Remark 5.2. If the operator representation (3.2) of $n$ is $n(z)=\left((A-z)^{-1} u, u\right)$ with the space $\mathcal{H}=\mathcal{H}(n)$, then, according to Theorem 3.1 the operator representation of $n_{1}=\widehat{n}$ is given by

$$
\mathcal{H}_{1}^{\prime}=\mathcal{H} \ominus\{u\}, \quad A_{1}=\left.P_{\mathcal{H}_{1}^{\prime}} A\right|_{\mathcal{H}_{1}^{\prime}}, \quad u_{1}=\frac{P_{\mathcal{H}_{1}^{\prime}} A u}{\|u\|},
$$

the operator representation of $n_{2}=\widehat{n}_{1}$ by

$$
\mathcal{H}_{2}^{\prime}=\mathcal{H} \ominus\{u, A u\}, \quad A_{2}=\left.P_{\mathcal{H}_{2}^{\prime}} A\right|_{\mathcal{H}_{2}^{\prime}}, \quad u_{2}=\frac{P_{\mathcal{H}_{2}^{\prime}} A^{2} u}{\|u\|\left\|u_{1}\right\|},
$$

and, via induction, the operator representation of $n_{p}=\widehat{n}_{p-1}$ by

$$
\mathcal{H}_{p}^{\prime}=\mathcal{H} \ominus\left\{u, A u, \cdots, A^{p-1} u\right\}, \quad A_{p}=\left.P_{\mathcal{H}_{p}^{\prime}} A\right|_{\mathcal{H}_{p}^{\prime}}, \quad u_{p}=\frac{P_{\mathcal{H}_{p}^{\prime}} A^{p} u}{\|u\|\left\|u_{1}\right\| \ldots\left\|u_{p-1}\right\|} .
$$

Note that $\|u\|=\sqrt{s_{0}}$ and, by Theorem $5.1,\left\|u_{j}\right\|=b_{j-1}, j=1, \ldots, p$.

Proof of Theorem 5.1. With the matrix (5.2), the equation $(A-z) x=u$ becomes

$$
\begin{aligned}
\left(A_{0}-z\right) x_{1}+b_{p-1}\left(x_{2}, e_{p}\right) e_{p-1} & =u, \\
b_{p-1}\left(x_{1}, e_{p-1}\right) e_{p}+(D-z) x_{2} & =0,
\end{aligned}
$$

where $x$ is written as $x=\left(\begin{array}{ll}x_{1} & x_{2}\end{array}\right)^{\top}$ according to the decomposition (5.1). The second equation implies

$$
x_{2}=-b_{p-1}\left(x_{1}, e_{p-1}\right)(D-z)^{-1} e_{p} .
$$

We insert this into (5.6) and apply $\left(A_{0}-z\right)^{-1}$ to get

$$
x_{1}=\left(A_{0}-z\right)^{-1} u+b_{p-1}^{2}\left(x_{1}, e_{p-1}\right)\left((D-z)^{-1} e_{p}, e_{p}\right)\left(A_{0}-z\right)^{-1} e_{p-1} .
$$

Now take the inner product with $e_{p-1}$ and solve the obtained equation for $\left(x_{1}, e_{p-1}\right)$ :

$$
\left(x_{1}, e_{p-1}\right)=\frac{\left(\left(A_{0}-z\right)^{-1} u, e_{p-1}\right)}{1-b_{p-1}^{2}\left((D-z)^{-1} e_{p}, e_{p}\right)\left(\left(A_{0}-z\right)^{-1} e_{p-1}, e_{p-1}\right)} .
$$

Observing that $n(z)=\left((A-z)^{-1} u, u\right)=\left(x_{1}, u\right)$, the relation (5.7) yields (5.4). To prove (5.5), denote by $P^{\prime}$ the orthogonal projection onto $\mathcal{H}_{p}^{\prime}$ in (5.1). If $k=1$, then $u \in \operatorname{dom} A^{p+1}$ is equivalent to $v:=A^{p} u \in \operatorname{dom} A$. Since $D$ is the only 
entry in the matrix of (5.2) which is possibly unbounded ( $B$ and $\widetilde{B}$ are even onedimensional), $v \in A$ is equivalent to the fact that the non-zero component $P^{\prime} v$, which is a multiple of $e_{p}$, belongs to $\operatorname{dom} D$. If $k=2$ we observe that

$$
A^{2}=\left(\begin{array}{cc}
A_{0}^{2}+\widetilde{B} B & A_{0} \widetilde{B}+\widetilde{B} D \\
B A_{0}+D B & B \widetilde{B}+D^{2}
\end{array}\right) .
$$

Since $e_{p} \in \operatorname{dom} D$ the operators $D B$ and $\widetilde{B} D$ and hence all the entries in the matrix representation of $A^{2}$ except possibly $D^{2}$ are bounded. Now $u \in \operatorname{dom} A^{p+2}$ is equivalent to $v=A^{p} u \in \operatorname{dom} A^{2}$, and hence, by (5.8), $P^{\prime} v \in \operatorname{dom} D^{2}$. The claim for arbitrary $k$ follows by induction.

The last claim of the theorem for $j=1$ follows immediately from (5.5) and the first equivalence in (4.1). For $j=2,3$ we also use the equivalences in (4.1). A simple calculation yields

$$
(A-z)^{-1}=\left(\begin{array}{cc}
R_{11}(z) & R_{12}(z) \\
R_{12}\left(z^{*}\right)^{*} & R_{22}(z)
\end{array}\right)
$$

with

$$
\begin{gathered}
R_{11}(z)=S_{1}(z)^{-1}, \quad R_{12}(z)=-b_{p-1}\left((D-z)^{-1} \cdot, e_{p}\right) S_{1}(z)^{-1} e_{p-1}, \\
R_{22}(z)=(D-z)^{-1}+b_{p-1}^{2}\left(S_{1}(z)^{-1} e_{p-1}, e_{p-1}\right)\left((D-z)^{-1} \cdot, e_{p}\right)(D-z)^{-1} e_{p},
\end{gathered}
$$

where $S_{1}(z):=A_{0}-z-b_{p-1}^{2}\left((D-z)^{-1} e_{p}, e_{p}\right)\left(\cdot, e_{p-1}\right) e_{p-1}$, the first Schur complement. It is easy to see that for $f, g \in \mathcal{H}_{p}$ we have

$$
\lim _{y \rightarrow \infty} \mathrm{i} y\left(S_{1}(\mathrm{i} y)^{-1} f, g\right)=-(f, g) .
$$

Now we observe the relation

$$
A(A-z)^{-1}=\left(\begin{array}{cc}
A_{0} R_{11}(z)+\widetilde{B} R_{12}\left(z^{*}\right)^{*} & A_{0} R_{12}(z)+\widetilde{B} R_{22}(z) \\
B R_{11}(z)+D R_{12}\left(z^{*}\right)^{*} & B R_{12}(z)+D R_{22}(z)
\end{array}\right)
$$

and the fact that for $z=\mathrm{i} y, y \rightarrow \infty$, for example $z A_{0} R_{11}(z)=z A_{0} S_{1}(z)$ has a limit,

$$
\begin{aligned}
z A_{0} R_{12}(z) & =-z b_{p-1}\left((D-z)^{-1} \cdot, e_{p}\right) A_{0} S_{1}(z)^{-1} e_{p-1}=\mathrm{o}(1), \\
z D R_{22}(z) & =z D(D-z)^{-1}+z b_{p-1}^{2}\left(S_{1}(z)^{-1} e_{p}, e_{p}\right)\left((D-z)^{-1} \cdot, e_{p}\right) D(D-z)^{-1} e_{p} \\
& =z D(D-z)^{-1}+\mathrm{o}(1),
\end{aligned}
$$

etc. These relations imply for example with $v=A^{p+k} u$

$$
z\left(A(A-z)^{-1} v, v\right)=z\left(D(D-z)^{-1} P^{\prime} v, P^{\prime} v\right)+\mathrm{O}(1) .
$$

Since $P^{\prime} v \in \operatorname{span}\left\{e_{p}, e_{p+1}, \ldots, e_{p+k}\right\}, e_{p}, e_{p+1}, \ldots, e_{p+k-1} \in \operatorname{dom} D$ and hence

$$
z\left(D(D-z)^{-1} x^{\prime}, x^{\prime}\right)=\mathrm{O}(1) \text { for } x^{\prime} \in \operatorname{span}\left\{e_{p}, e_{p+1}, \ldots, e_{p+k}\right\},
$$

and since $P^{\prime} v$ has a non-zero component in the direction of $e_{p+k}$ the claim follows from (4.1). 


\section{Representation of the transformation matrix by orthogonal polynomials}

The $2 \times 2$ matrix function which generates the fractional linear transformation (5.4) we denote in the following by $V$ :

$$
V(z):=\frac{1}{a(z)}\left(\begin{array}{cc}
\check{r}(z) & -a_{00}(z) \\
a_{11}(z) & -1
\end{array}\right)
$$

In this section we express $V$ by the polynomials of first and second kind. To this end, the elements of $\mathcal{H}_{p}$ are considered as column vectors with respect to the basis $e_{0}, e_{1}, \ldots, e_{p-1}$.

First we solve the equation $\left(A_{0}-z\right) x=e_{p-1}$ in $\mathcal{H}_{p}$. With the Jacobi matrix $\mathcal{A}_{0}$ from (4.7) this equation becomes

$$
\mathcal{A}_{0} x-z x=e_{p-1},
$$

or

$$
\left(\mathcal{A}_{0}-z\right)\left(\begin{array}{c}
\xi_{1} \\
\vdots \\
\xi_{p-1} \\
\xi_{p}
\end{array}\right)=\left(\begin{array}{c}
0 \\
\vdots \\
0 \\
1
\end{array}\right) .
$$

According to the definition of the orthogonal polynomials of first kind, the solution of the system with the 1 in the last component of the vector on the right hand side replaced by $-b_{p-1} e_{p}(z)$ is the vector with components $e_{0}(z), e_{1}(z), \ldots, e_{p-1}(z)$. It follows that

$$
\xi_{j}=-\frac{e_{j-1}(z)}{b_{p-1} e_{p}(z)}, \quad j=1,2, \ldots, p,
$$

and hence

$$
\begin{aligned}
& \left(\left(A_{0}-z\right)^{-1} e_{p-1}, e_{p-1}\right)=\left(x, e_{p-1}\right)=-\frac{e_{p-1}(z)}{b_{p-1} e_{p}(z)}, \\
& \left(\left(A_{0}-z\right)^{-1} e_{p-1}, e_{0}\right)=\left(x, e_{0}\right)=-\frac{e_{0}(z)}{b_{p-1} e_{p}(z)},
\end{aligned}
$$

that is,

$$
a_{11}(z)=-\frac{e_{p-1}(z)}{b_{p-1} e_{p}(z)}, \quad a(z)=-\frac{1}{b_{p-1} e_{p}(z)} .
$$

Next we solve the equation $\left(A_{0}-z\right) x=u=\sqrt{s_{0}} e_{0}$. As above, in matrix form it becomes

$$
\left(\mathcal{A}_{0}-z\right)\left(\begin{array}{c}
\xi_{1} \\
\xi_{2} \\
\vdots \\
\xi_{p}
\end{array}\right)=\left(\begin{array}{c}
\sqrt{s_{0}} \\
0 \\
\vdots \\
0
\end{array}\right)
$$


According to the definition of the polynomials of the second kind and because of $b_{0} d_{1}(z)=\sqrt{s_{0}}$ we have

$$
\left(\mathcal{A}_{0}-z\right)\left(\begin{array}{c}
0 \\
d_{1}(z) \\
\vdots \\
d_{p-2}(z) \\
d_{p-1}(z)
\end{array}\right)=\left(\begin{array}{c}
\sqrt{s_{0}} \\
0 \\
\vdots \\
0 \\
-b_{p-1} d_{p}(z)
\end{array}\right)=\left(\begin{array}{c}
\sqrt{s_{0}} \\
0 \\
\vdots \\
0 \\
0
\end{array}\right)-b_{p-1} d_{p}(z)\left(\begin{array}{c}
0 \\
0 \\
\vdots \\
0 \\
1
\end{array}\right) .
$$

It follows that

$$
\left(\begin{array}{c}
\xi_{1} \\
\xi_{2} \\
\vdots \\
\xi_{p}
\end{array}\right)=\left(\mathcal{A}_{0}-z\right)^{-1}\left(\begin{array}{c}
\sqrt{s_{0}} \\
0 \\
\vdots \\
0
\end{array}\right)=\left(\begin{array}{c}
0 \\
d_{1}(z) \\
\vdots \\
d_{p-1}(z)
\end{array}\right)-\frac{d_{p}(z)}{e_{p}(z)}\left(\begin{array}{c}
e_{0}(z) \\
e_{1}(z) \\
\vdots \\
e_{p-1}(z)
\end{array}\right)
$$

and hence

$$
a_{00}(z)=\left(\left(A_{0}-z\right)^{-1} u, u\right)=-\frac{d_{p}(z)}{e_{p}(z)} .
$$

Inserting the expressions from (6.2) and (6.3) into (5.4) and observing the relation (4.15) we find that $V$ can be written as

$$
V(z)=\left(\begin{array}{cc}
-d_{p-1}(z) & -b_{p-1} d_{p}(z) \\
e_{p-1}(z) & b_{p-1} e_{p}(z)
\end{array}\right)
$$

and hence we obtain the following theorem.

Theorem 6.1. If, for some integer $p \geq 1$, the Nevanlinna function $n$ satisfies one of the assumptions $\left(j_{p}\right), j \in\{1,2,3\}$, and $D_{p} \neq 0$ then the following relation holds:

$$
n(z)=\left((A-z)^{-1} u, u\right)=-\frac{d_{p-1}(z) n_{p}(z)+b_{p-1} d_{p}(z)}{e_{p-1}(z) n_{p}(z)+b_{p-1} e_{p}(z)},
$$

where $n_{p}(z)=\left((D-z)^{-1} u_{p}, u_{p}\right), u_{p}=b_{p-1} e_{p}$.

Remark 6.2. (i) Using (4.12) and (4.6) we obtain from (6.4) with $p=1$ :

$$
n(z)=-\frac{s_{0}}{z-\frac{s_{1}}{s_{0}}+n_{1}(z)}
$$

and hence, because $n_{1}(z)=\mathrm{o}(1), n_{1}$ is the Schur transform of $n: n_{1}=\widehat{n}$. For $p \geq 2$ we obtain from (6.4) and (6.4) with $p$ replaced by $p-1$ and with the help of (4.5) and (4.15) that

$$
n_{p-1}(z)=-\frac{b_{p-2}^{2}}{z-a_{p-1}+n_{p}(z)},
$$

hence

$$
n_{p-1}(z)=-\frac{b_{p-2}^{2}}{z}-\frac{a_{p-1} b_{p-2}^{2}}{z^{2}}+\mathrm{o}\left(\frac{1}{z^{2}}\right)
$$


and $n_{p}=\widehat{n}_{p-1}$.

(ii) From (6.4), (4.17), 4.18), and (4.20), we obtain

$$
\widehat{n}(z)=-\frac{s_{0}}{n(z)}-\left(z-a_{0}\right)=-\frac{\widehat{d}_{p-2}(z) n_{p}(z)+\widehat{b}_{p-2} \widehat{d}_{p-1}(z)}{\widehat{e}_{p-2}(z) n_{p}(z)+\widehat{b}_{p-2} \widehat{e}_{p-1}(z)} .
$$

Since, according to Theorem 4.2, $\widehat{d}_{k}$ and $\widehat{e}_{k}$ are the polynomials of first and second kind associated with $\widehat{n}$, this formula implies that the function $n_{p}$ is the $p-1$-st Schur transform of $\widehat{n}$.

The $2 \times 2$ matrix polynomial $V$, which generates the fractional linear transformation (6.4), has the property

$$
\operatorname{det} V(z)=b_{p-1}\left(d_{p}(z) e_{p-1}(z)-e_{p}(z) d_{p-1}(z)\right)=1 .
$$

With

$$
J=\left(\begin{array}{cc}
0 & 1 \\
-1 & 0
\end{array}\right)
$$

$V$ is $J$-unitary on the real line, that is,

$$
V(z) J V(z)^{*}=J \quad z \in \mathbb{R} .
$$

Therefore $V(z)^{-1}$ exists for all $z \in \mathbb{C}$ and we can form the polynomial matrix function

$$
V_{0}(z)=V(z) V(0)^{-1}=\left(\begin{array}{cc}
p_{1}^{(p)}(z) & p_{0}^{(p)}(z) \\
q_{1}^{(p)}(z) & q_{0}^{(p)}(z)
\end{array}\right)
$$

with

$$
\begin{aligned}
& p_{0}^{(p)}(z)=b_{p-1}\left(d_{p}(z) d_{p_{-1}}(0)-d_{p-1}(z) d_{p}(0)\right), \\
& p_{1}^{(p)}(z)=b_{p-1}\left(d_{p}(z) e_{p-1}(0)-d_{p-1}(z) e_{p}(0)\right), \\
& q_{0}^{(p)}(z)=b_{p-1}\left(e_{p-1}(z) d_{p}(0)-e_{p}(z) d_{p-1}(0)\right), \\
& q_{1}^{(p)}(z)=b_{p-1}\left(e_{p-1}(z) e_{p}(0)-e_{p}(z) e_{p-1}(0)\right) .
\end{aligned}
$$

A straightforward calculation leads to the relation

$$
n(z) \equiv\left((A-z)^{-1} u, u\right)=\frac{p_{1}^{(p)}(z) h_{p}(z)+p_{0}^{(p)}(z)}{q_{1}^{(p)}(z) h_{p}(z)+q_{0}^{(p)}(z)},
$$

where

$$
h_{p}(z)=-\frac{d_{p-1}(0) n_{p}(z)+b_{p-1} d_{p}(0)}{e_{p-1}(0) n_{p}(z)+b_{p-1} e_{p}(0)} .
$$


With relation (4.14), the following formulas can be obtained, compare [1, I.2.4]:

$$
\begin{aligned}
& p_{0}^{(p)}(z)=z \sum_{k=0}^{p-1} d_{k}(z) d_{k}(0), \\
& p_{1}^{(p)}(z)=1+z \sum_{k=0}^{p-1} d_{k}(z) e_{k}(0), \\
& q_{0}^{(p)}(z)=1-z \sum_{k=0}^{p-1} e_{k}(z) d_{k}(0), \\
& q_{1}^{(p)}(z)=-z \sum_{k=0}^{p-1} e_{k}(z) e_{k}(0) .
\end{aligned}
$$

\section{Transformation by means of a $u$-resolvent matrix}

Given again a function $n \in \mathbf{N}_{0}$ with one of the properties $\left(j_{p}\right), j=1,2,3$. Besides the decomposition (5.1) we consider the decomposition

$$
\mathcal{H}(n)=\mathcal{H}_{p+1} \oplus \mathcal{H}^{\prime \prime}, \quad \mathcal{H}_{p+1}=\operatorname{span}\left\{\mathcal{H}_{p}, e_{p}\right\}=\operatorname{span}\left\{e_{0}, e_{1}, \ldots, e_{p}\right\},
$$

and in the space $\mathcal{H}_{p+1}$ the restriction

$$
S:=\left.A\right|_{\mathcal{H}_{p}}=\left(\begin{array}{c}
A_{0} \\
B
\end{array}\right) .
$$

This restriction is a non-densely defined symmetric operator in $\mathcal{H}_{p+1}$ with defect index $(1,1)$, and, evidently, the given function $n=\left((A-z)^{-1} u, u\right)$ is one of the $u$-resolvents of this operator $S$. Hence $n$ can be represented as a fractional linear transformation of some function $g \in \mathbf{N}_{0}$ by means of the $u$-resolvent matrix $W=$ $\left(w_{k \ell}\right)_{k, \ell=1}^{2}$ of $S$ :

$$
n(z)=\frac{w_{11}(z) g(z)+w_{12}(z)}{w_{21}(z) g(z)+w_{22}(z)} .
$$

Such a $u$-resolvent matrix $W$ can easily be calculated. To this end we fix a self-adjoint extension of $S$ in $\mathcal{H}_{p+1}$, which means that we fix some $\gamma \in \mathbb{R}$ in the right lower corner of the matrix representation of $S$ with respect to the basis $e_{0}, e_{1}, \ldots, e_{p}$ of $\mathcal{H}_{p+1}$. Denote this matrix or self-adjoint extension of $S$ in $\mathcal{H}_{p+1}$ by $A_{0, \gamma}$ :

$$
A_{0, \gamma}=\left(\begin{array}{cc}
A_{0} & \widetilde{B} \\
B & \gamma
\end{array}\right)
$$

According to [14 this $u$-resolvent matrix $W$ is given by the formula

$$
W(z)=\frac{1}{\left(u, \varphi\left(z^{*}\right)\right)}\left(\begin{array}{cc}
\left(R_{z}^{\gamma} u, u\right) & \left(R_{z}^{\gamma} u, u\right) Q(z)-\left(u, \varphi\left(z^{*}\right)\right)(\varphi(z), u) \\
1 & Q(z)
\end{array}\right),
$$


where $R_{z}^{\gamma}=\left(A_{0, \gamma}-z\right)^{-1}, \varphi(z)$ is a defect function of $S$ corresponding to the selfadjoint extension $A_{0, \gamma}$, and $Q$ is the corresponding $Q$-function. An easy calculation yields

$$
\left(R_{z}^{\gamma} u, u\right)=\left(\left(A_{0, \gamma}-z\right)^{-1} u, u\right)=a_{00}(z)-\frac{b_{p-1}^{2} a(z)^{2}}{\Delta(z)},
$$

where

$$
\Delta(z)=z-\gamma+b_{p-1}^{2} a_{11}(z) .
$$

Since $S=\left.A_{0, \gamma}\right|_{\mathcal{H}_{p}}$ and hence, in terms of linear relations,

$$
S^{*}=\left\{\left\{x, A_{0, \gamma} x+\lambda e_{p}\right\} \mid x \in \mathcal{H}_{p+1}, \lambda \in \mathbb{C}\right\},
$$

it is easy to check that for $\varphi(z)$ with $\{\varphi(z), z \varphi(z)\} \in S^{*}$ we can choose

$$
\varphi(z)=\left(A_{0, \gamma}-z\right)^{-1} e_{p}=\frac{-1}{\Delta(z)}\left(\begin{array}{c}
-b_{p-1}\left(A_{0}-z\right)^{-1} e_{p-1} \\
1
\end{array}\right),
$$

and then the $Q$-function, which is the solution (up to a real additive constant) of the equation

$$
\frac{Q(z)-Q(\zeta)^{*}}{z-\zeta^{*}}=(\varphi(z), \varphi(\zeta))
$$

becomes

$$
Q(z)=\frac{-1}{\Delta(z)} .
$$

Inserting these expressions into $W$ from (7.2) we find

$$
W(z)=\frac{\Delta(z)}{b_{p-1} a(z)}\left(\begin{array}{cc}
a_{00}(z)-b_{p-1}^{2} \frac{a(z)^{2}}{\Delta(z)} & -\frac{a_{00}(z)}{\Delta(z)} \\
1 & \frac{-1}{\Delta(z)}
\end{array}\right) .
$$

Observe that $W(z)$ is $J$-unitary on the real line. Next we establish the connection between the matrix functions $V$ from (6.1) and $W$ from (7.2), in fact we find a simple expression for $V^{-1} W$. We have

$$
V(z)^{-1}=\frac{1}{a(z)}\left(\begin{array}{cc}
-1 & a_{00}(z) \\
-a_{11}(z) & a_{00}(z) a_{11}(z)-a(z)^{2}
\end{array}\right) .
$$

Multiplying this matrix from the right by $W(z)$ from (7.3) we obtain

$$
V(z)^{-1} W(z)=-\left(\begin{array}{cc}
-b_{p-1} & 0 \\
\frac{z-\gamma}{b_{p-1}} & -\frac{1}{b_{p-1}}
\end{array}\right) .
$$

Theorem 7.1. If the function $n \in \mathbf{N}_{0}$ has one of the properties $\left(j_{p}\right), j \in\{1,2,3\}$, then the matrix functions $V$ from (6.1) and $W$ from (7.2) are connected by the 
relation (7.4). Therefore for the Nevanlinna functions $n_{p}$ in (5.4) and $g$ in (7.1) the following relation holds:

$$
n_{p}(z)=-\frac{b_{p-1}^{2}}{z-\gamma-\frac{1}{g(z)}} .
$$

If $1 / g(z)=\mathrm{o}(1)$, then formula (7.5) implies that $n_{p}$ admits the asymptotic expansion

$$
n_{p}(z)=-\frac{b_{p-1}^{2}}{z}-\frac{\gamma b_{p-1}^{2}}{z^{2}}+\mathrm{o}\left(\frac{1}{z^{2}}\right)
$$

and $-1 / g$ is its Schur transform: $-1 / g=\widehat{n}_{p}$. Hence the number $\gamma$, which defines the self-adjoint extension of $S$, corresponds to the number $a_{p}$.

Remark 7.2. If instead of a self-adjoint operator extension $A_{0, \gamma}$ of $S$ we choose the (multi-valued) self-adjoint relation extension of S:

$$
A_{0, \infty}=S+\operatorname{span}\left\{0, e_{p}\right\}=A_{0}+\operatorname{span}\left\{0, e_{p}\right\},
$$

then we obtain

$$
R_{z}^{\infty}=\left(A_{0, \infty}-z\right)^{-1}=\left(A_{0}-z\right)^{-1} P
$$

where $P$ is the orthogonal projection in $\mathcal{H}_{p+1}$ onto $\mathcal{H}_{p}$,

$$
\varphi(z)=\left(\begin{array}{c}
-b_{p-1}\left(A_{0}-z\right)^{-1} e_{p-1} \\
1
\end{array}\right), \quad Q(z)=z+b_{p-1}^{2} a_{11}(z)
$$

so that

$$
\begin{gathered}
W(z)=-\frac{1}{b_{p-1}^{2} a(z)}\left(\begin{array}{cc}
a_{00}(z) & a_{00}\left(z+b_{p-1}^{2} a_{11}(z)\right)-b_{p-1}^{2} a(z)^{2} \\
1 & z+b_{p-1}^{2} a_{11}(z)
\end{array}\right), \\
V(z)^{-1} W(z)=\left(\begin{array}{cc}
0 & -b_{p-1} \\
\frac{1}{b_{p-1}} & \frac{z}{b_{p-1}}
\end{array}\right),
\end{gathered}
$$

and instead of (7.5) we have

$$
n_{p}(z)=-\frac{b_{p-1}^{2}}{z+g(z)} .
$$

Thus if $g(z)=\mathrm{o}(1)$, then $n_{p}$ has the asymptotic expansion

$$
n_{p}(z)=-\frac{b_{p-1}^{2}}{z}+\mathrm{o}\left(\frac{1}{z^{2}}\right)
$$

and $g$ is the Schur transform of $n_{p}: g=\widehat{n}_{p}$.

A more explicit form of the resolvent matrix $W$ from (7.2) can be obtained following [14] and 22. To this end we decompose the space $\mathcal{H}_{p+1}$ as

$$
\mathcal{H}_{p+1}=\operatorname{ran}(S-z) \dot{+} \operatorname{span} u, \quad z \in \mathbb{C}, a(z) \neq 0,
$$


( $\dot{+}$ stands for direct sum) and denote for $y \in \mathcal{H}_{p+1}$ by $\mathrm{P}(z) y$ the coefficient of $u$ in the corresponding decomposition of $y$ :

$$
y=(S-z) x+(\mathrm{P}(z) y) u
$$

with some $x \in \operatorname{dom} S=\mathcal{H}_{p}$. Further, define $\mathrm{Q}(z) y=\left((S-z)^{-1}(y-(\mathrm{P}(z) y) u), u\right)$. Then, according to [14, the resolvent matrix can be chosen to be

$$
W^{0}(z)=I_{2}+z\left(\begin{array}{c}
\mathrm{Q}(z) \\
-\mathrm{P}(z)
\end{array}\right)\left(\mathrm{Q}(0)^{*}-\mathrm{P}(0)^{*}\right) J, \quad J=\left(\begin{array}{cc}
0 & 1 \\
-1 & 0
\end{array}\right) .
$$

We derive an explicit expression for $W^{0}(z):=W(z) W(0)^{-1}$, following [2]. To this end, for the vectors and operators we use matrix representations with respect to the basis $\mathbf{t}_{0}(=u), \mathbf{t}_{1}, \ldots, \mathbf{t}_{p}$. Recall that $S_{p}$ is the Gram matrix associated with this basis. We denote by $\mathfrak{S}$ the $(p+1) \times(p+1)$-matrix

$$
\mathfrak{S}=\left(\begin{array}{ccccc}
0 & \cdots & \cdots & \cdots & 0 \\
1 & 0 & \cdots & \cdots & 0 \\
0 & 1 & 0 & \cdots & \vdots \\
\vdots & & \ddots & \ddots & \vdots \\
0 & \cdots & 0 & 1 & 0
\end{array}\right)
$$

and by $e$ the first column in the $(p+1) \times(p+1)$ identity matrix. Then $S$ and $u$ correspond to $\left.\mathfrak{S}\right|_{\mathbb{C}^{p}+\{0\}}$ and $e$. First we apply the operator $\left(I-z \mathfrak{S}^{*}\right)^{-1}$ to (7.6) and observe the relation

$$
e^{*}\left(I-z \mathfrak{S}^{*}\right)^{-1}(\mathfrak{S}-z) x=0, \quad x \in \mathbb{C}^{p} \dot{+}\{0\} .
$$

It follows that

$$
e^{*}\left(I-z \mathfrak{S}^{*}\right)^{-1} y=(\mathrm{P}(z) y) e^{*}\left(I-z \mathfrak{S}^{*}\right)^{-1} u=\mathrm{P}(z) y .
$$

Further, observing that $e^{*} S_{p}=\left(\begin{array}{llll}s_{0} & s_{1} & \cdots & s_{p}\end{array}\right)$ we obtain

$$
\begin{aligned}
& \mathrm{Q}(z) y=\left((S-z)^{-1}(y-(\mathrm{P}(z) y) u), u\right) \\
& =e^{*} S_{p}\left((\mathfrak{S}-z)^{-1} y-(\mathfrak{S}-z)^{-1} e\left(e^{*}\left(I-z \mathfrak{S}^{*}\right)^{-1} y\right)\right) \\
& =\left(\begin{array}{llll}
s_{0} & s_{1} & \cdots & s_{p}
\end{array}\right)\left((\mathfrak{S}-z)^{-1}\left(I-z \mathfrak{S}^{*}\right)-(\mathfrak{S}-z)^{-1} e e^{*}\right)\left(I-z \mathfrak{S}^{*}\right)^{-1} y \\
& =\left(\begin{array}{llll}
s_{0} & s_{1} & \cdots & s_{p}
\end{array}\right) \mathfrak{S}^{*}\left(I-z \mathfrak{S}^{*}\right)^{-1} y \\
& =\left(\begin{array}{lllll}
0 & s_{0} & s_{1} & \cdots & s_{p-1}
\end{array}\right)\left(I-z \mathfrak{S}^{*}\right)^{-1} y,
\end{aligned}
$$

where for the second last equality sign we have used that

$$
(\mathfrak{S}-z)^{-1}\left(I-z \mathfrak{S}^{*}\right)-(\mathfrak{S}-z)^{-1} e e^{*}=\mathfrak{S}^{*} .
$$

Together with (7.8) we find

$$
\left(\begin{array}{c}
\mathrm{Q}(z) \\
-\mathrm{P}(z)
\end{array}\right)=\left(\begin{array}{ccccc}
0 & s_{0} & s_{1} & \cdots & s_{p-1} \\
-1 & 0 & 0 & \cdots & 0
\end{array}\right)\left(I-z \mathfrak{S}^{*}\right)^{-1}
$$


and (7.7) becomes

$$
W^{0}(z)=I_{2}+z\left(\begin{array}{cccc}
0 & s_{0} & \cdots & s_{p-1} \\
-1 & 0 & \cdots & 0
\end{array}\right)\left(I-z \mathfrak{S}^{*}\right)^{-1} S_{p}^{-1}\left(\begin{array}{cc}
0 & -1 \\
s_{0} & 0 \\
\vdots & \vdots \\
s_{p-1} & 0
\end{array}\right)\left(\begin{array}{cc}
0 & 1 \\
-1 & 0
\end{array}\right) .
$$

\section{Reproducing kernel spaces: reduction via resolvent invariant subspaces}

In this section we start from the operator representation of the Nevanlinna function $n$ in the corresponding reproducing kernel space $\mathcal{L}(n)$ with kernel

$$
L_{n}(z, w)=\frac{n(z)-n(w)^{*}}{z-w^{*}}, \quad z, \zeta \in \mathbb{C} \backslash \mathbb{R},
$$

see Section 2 (3). The operator $A$ is introduced via its resolvent $(A-z)^{-1}$ which is the difference-quotient operator $R_{z}$ defined by

$$
\left(R_{z} f\right)(\zeta)=\frac{f(\zeta)-f(z)}{\zeta-z}, \quad f \in \mathcal{L}(n) .
$$

If $n$ satisfies one of the assumptions $\left(j_{p}\right)$, then, by [3, Lemma 5.1], the functions

$$
f_{0}(\zeta)=n(\zeta), f_{1}(\zeta)=\zeta n(\zeta)+s_{0}, \ldots, f_{p}(\zeta)=\zeta^{p} n(\zeta)+\zeta^{p-1} s_{0}+\cdots+s_{p-1}
$$

all belong to $\mathcal{L}(n)$ and

$$
\left\langle f_{k}, f_{j}\right\rangle_{\mathcal{L}(n)}=s_{j+k}, \quad j, k=0,1, \ldots, p .
$$

In particular, $u:=n \in \mathcal{L}(n)$, and by the reproducing property of the kernel $L_{n}$ we have

$$
n(z)=\left((A-z)^{-1} u, u\right)_{\mathcal{L}(n)} .
$$

By $\mathcal{U}_{J}$ we denote the class of all $2 \times 2$ matrix polynomials $\Theta$ which are $J$ unitary on $\mathbb{R}$ and for which the kernel

$$
K_{\Theta}(z, w)=\frac{J-\Theta(z) J \Theta(w)^{*}}{z-w^{*}}
$$

is non-negative. The reproducing kernel Hilbert space with this kernel will be denoted by $\mathcal{H}(\Theta)$; its elements are 2 -vector functions. The matrix polynomials $V$ and $W$ considered in the previous sections belong to $\mathcal{U}_{J}$ : this follows from the Christoffel-Darboux formulas (4.14) for $V$ and from (7.4) for $W$. Note that if $\Theta$ belongs to $\mathcal{U}_{J}$, then $\operatorname{det} \Theta(z) \equiv c$, where is $c$ is a unimodular complex number, because the determinant $\operatorname{det} \Theta(z)$ is a non-vanishing polynomial in $z$.

The following theorem was proved in [4, Theorem 8.1], even in an indefinite setting. 
Theorem 8.1. Let $n \in \mathbf{N}_{0}$ and suppose that there exists a matrix polynomial

$$
\Theta=\left(\begin{array}{ll}
a & b \\
c & d
\end{array}\right) \in \mathcal{U}_{\ell}
$$

such that the mapping

$$
\mathbf{u} \longrightarrow\left(\begin{array}{ll}
1 & -n
\end{array}\right) \mathbf{u}
$$

is an isometry from $\mathcal{H}(\Theta)$ into $\mathcal{L}(n)$. Define the function $\check{n}$ by

$$
n(z)=\frac{a(z) \check{n}(z)+b(z)}{c(z) \check{n}(z)+d(z)} .
$$

Then the following statements hold.

(i) $\check{n}$ is Nevanlinna function.

(ii) The mapping $g \mapsto f$ :

$$
f(\zeta)=(a(\zeta)-n(\zeta) c(\zeta)) g(\zeta)
$$

is an isometry from $\mathcal{L}(\check{n})$ into $\mathcal{L}(n)$.

(iii) We have

$$
\mathcal{L}(n)=(1 \quad-n) \mathcal{H}(\Theta) \oplus(a-n c) \mathcal{L}(\check{n})
$$

and the mapping

$$
W: \mathcal{L}(n) \ni f \mapsto\left(\begin{array}{c}
\mathbf{u} \\
g
\end{array}\right) \in\left(\begin{array}{c}
\mathcal{H}(\Theta) \\
\mathcal{L}(\check{n})
\end{array}\right)
$$

where $f, \mathbf{u}$, and $g$ are connected by the relation

$$
f(\zeta)=(1-n(\zeta)) \mathbf{u}(\zeta)+(a(\zeta)-n(\zeta) c(\zeta)) g(\zeta),
$$

is a unitary mapping from $\mathcal{L}(n)$ onto $\mathcal{H}(\Theta) \oplus \mathcal{L}(\check{n})$.

(iv) The mapping $W R_{z} W^{*}$ is of the form

$$
W R_{z} W^{*}=\left(\begin{array}{ll}
R_{11}(z) & R_{12}(z) \\
R_{21}(z) & R_{22}(z)
\end{array}\right):\left(\begin{array}{l}
\mathcal{P}(\Theta) \\
\mathcal{L}(\check{n})
\end{array}\right) \rightarrow\left(\begin{array}{c}
\mathcal{P}(\Theta) \\
\mathcal{L}(\check{n})
\end{array}\right)
$$


with

$$
\begin{aligned}
& R_{11}(z)=R_{z}-\frac{1}{k(z)}\left(R_{z} \Theta\right)(\cdot)\left(\begin{array}{c}
\check{n}(z) \\
1
\end{array}\right)\left(\begin{array}{ll}
0 & 1
\end{array}\right) E_{z} \\
& =R_{z}-K_{\Theta}\left(\cdot, z^{*}\right)\left(\begin{array}{c}
1 \\
-n(z)
\end{array}\right)\left(\begin{array}{ll}
0 & 1
\end{array}\right) E_{z}, \\
& R_{12}(z)=\frac{1}{k(z)}\left(R_{z} \Theta\right)(\cdot)\left(\begin{array}{c}
d(z) \\
-c(z)
\end{array}\right) E_{z} \\
& =-(a(z)-n(z) c(z)) K_{\Theta}\left(\cdot, z^{*}\right)\left(\begin{array}{l}
0 \\
1
\end{array}\right) E_{z}, \\
& R_{21}(z)=-\frac{1}{k(z)}\left(R_{z} \check{n}\right)(\cdot)\left(\begin{array}{ll}
0 & 1
\end{array}\right) E_{z} \\
& =-\frac{1}{k(z)} L_{\check{n}}\left(\cdot, z^{*}\right)\left(\begin{array}{ll}
0 & 1
\end{array}\right) E_{z}, \\
& R_{22}(z)=R_{z}-\frac{c(z)}{k(z)}\left(R_{z} \check{n}\right)(\cdot) E_{z} \\
& =R_{z}-\frac{c(z)}{k(z)} L_{\check{n}}\left(\cdot, z^{*}\right) E_{z},
\end{aligned}
$$

where $R_{z}$ is the difference-quotient operator, $E_{z}$ is the operator of evaluation at the point $z$ on any reproducing kernel space, and

$$
k(z)=c(z) \check{n}(z)+d(z)=\frac{\operatorname{det} \Theta(z)}{a(z)-n(z) c(z)} .
$$

We mention that formula (8.3) corresponds to the relation (5.9) above.

A space of functions is called resolvent-invariant if it is invariant under the difference-quotient operator $R_{z}$ as defined in (8.1). In the following lemma, with a resolvent-invariant non-degenerate invariant subspace of a certain inner product space a $2 \times 2$ matrix function is associated.

Lemma 8.2. Let $\mathcal{M}$ be a finite dimensional resolvent-invariant space of 2-vector polynomials endowed with an inner product $\langle\cdot, \cdot\rangle$ such that

$$
\left\langle R_{z} f, g\right\rangle-\left\langle f, R_{w} g\right\rangle-\left(z-w^{*}\right)\left\langle R_{z} f, R_{w} g\right\rangle=g(w)^{*} J f(z),
$$

and let that $\mathcal{M}_{1}$ be a resolvent-invariant non-degenerate subspace of $\mathcal{M}$. Then there exists a $\Theta_{1} \in \mathcal{U}_{J}$ such that

(i) $\mathcal{M}_{1}=\mathcal{H}\left(\Theta_{1}\right)$,

(ii) $\mathcal{M}=\mathcal{H}\left(\Theta_{1}\right) \oplus \Theta_{1} \mathcal{N}$ where $\mathcal{N}=\Theta_{1}^{-1} \mathcal{M}_{1}^{\perp}$ is a resolvent-invariant space of 2-vector polynomials, for which the relation (8.4) holds if equipped with the inner product

$$
\left(\Theta_{1}^{-1} f, \Theta_{1}^{-1} g\right)_{\mathcal{N}}=\langle f, g\rangle, \quad f, g \in \mathcal{M}_{1}^{\perp} .
$$


Relation (8.4) is often called de Branges identity, see [7] and, for further references, [1]. That $\mathcal{N}$ consists of 2 -vector polynomials is due to fact that $\Theta_{1}^{-1}(z)=-J \Theta_{1}\left(z^{*}\right)^{*} J$ is a matrix polynomial. The other claims of the lemma follow from [6, Theorem 3.1].

Now we formulate and prove Theorem6.1 again in the context of reproducing kernel spaces.

Theorem 8.3. If, for some integer $p \geq 1$, the Nevanlinna function $n$ satisfies one of the assumptions $\left(j_{p}\right), j \in\{1,2,3\}$, and $D_{p} \neq 0$ then the following relation holds:

$$
n(z)=-\frac{d_{p-1}(z) \check{n}_{p}(z)+b_{p-1} d_{p}(z)}{e_{p-1}(z) \check{n}_{p}(z)+b_{p-1} e_{p}(z)}
$$

where $\check{n}_{p}$ is a Nevanlinna function such that $\check{n}_{p} \in \mathcal{L}\left(\check{n}_{p}\right)$, and

$$
\check{n}_{p}(z)=\left(R_{z} \check{n}_{p}, \check{n}_{p}\right)_{\mathcal{L}\left(\check{n}_{p}\right)} .
$$

Comparing (6.4) and (8.5) we find that $\check{n}_{p}(z)=n_{p}(z)$, the $p$-th element in the sequence obtained by applying the Schur transformation $p$ times starting with $n$.

Proof of Theorem 8.3 . Let $\mathcal{M}$ be the linear space spanned by the $p+12$-vector functions

$$
\mathbf{f}_{0}(\zeta)=\left(\begin{array}{c}
0 \\
-1
\end{array}\right), \mathbf{f}_{1}(\zeta)=\left(\begin{array}{c}
s_{0} \\
-\zeta
\end{array}\right), \ldots, \mathbf{f}_{p}(\zeta)=\left(\begin{array}{c}
s_{0} \zeta^{p-1}+\cdots+s_{p-1} \\
-\zeta^{p}
\end{array}\right)
$$

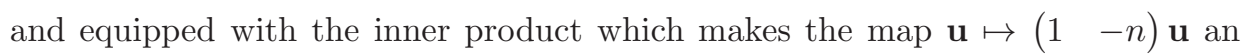
isometry from $\mathcal{M}$ into $\mathcal{L}(n)$, see (8.2). Note that if $n$ has the integral representation (2.2), the elements of $\mathcal{M}$ are of the form

$$
\left(\begin{array}{c}
\int_{-\infty}^{\infty}\left(R_{\zeta} f\right)(t) d \sigma(t) \\
-f(\zeta)
\end{array}\right)
$$

where $f$ is a polynomial of degree $\leq p$. Indeed, it suffices to show this for the basis elements of $\mathcal{M}$ : If $f(\zeta)=\zeta^{j}$, then

$$
R_{\zeta} f(t)=\frac{\zeta^{j}-t^{j}}{\zeta-t}=\zeta^{j-1}+t \zeta^{j-2}+\cdots+t^{j-2} \zeta+t^{j-1},
$$

and hence, on account of (1.3),

$$
\int_{-\infty}^{\infty}\left(R_{\zeta} f\right)(t) d \sigma(t)=s_{0} \zeta^{j-1}+s_{1} \zeta^{j-2}+\cdots+s_{p-2} \zeta+s_{p-1} .
$$

It follows that $\mathcal{M}$ is also spanned by the polynomial vectors

$$
\left(\begin{array}{c}
-d_{j} \\
e_{j}
\end{array}\right), \quad j=0,1, \ldots, p,
$$

where $e_{j}$ and $d_{j}$ are the polynomials of first and second kind associated with $n$, see (4.4) and (4.10). 
Let $\mathcal{M}_{p}$ be the space spanned by the first $p$ of the 2 -vector functions in (8.7). Since $S_{p-1}$ from (4.2) is a positive matrix, the space $\mathcal{M}_{p}$ is non-degenerate and

$$
\mathcal{M}=\mathcal{M}_{p} \oplus \operatorname{span}\left(\begin{array}{c}
-d_{p} \\
e_{p}
\end{array}\right) .
$$

As both $\mathcal{M}$ and $\mathcal{M}_{p}$ are resolvent-invariant spaces, by Lemma 8.2 we have that for some $\Theta_{1} \in \mathcal{U}_{J}$, which is normalized by $\Theta_{1}(0)=I_{2}$ (and hence $\operatorname{det} \Theta(z) \equiv 1$ ),

$$
\mathcal{M}_{p}=\mathcal{H}\left(\Theta_{1}\right), \quad \mathcal{M}=\mathcal{H}\left(\Theta_{1}\right) \oplus \Theta_{1} \mathcal{N} .
$$

Here $\mathcal{N}$ is a one-dimensional resolvent-invariant space, which, when equipped with the induced inner product, satisfies the de Branges identity and therefore is spanned by a constant $J$-neutral vector $\left(\begin{array}{ll}\alpha & \beta\end{array}\right)^{\top}$ such that

$$
\Theta_{1}(z)\left(\begin{array}{c}
\alpha \\
\beta
\end{array}\right)=b_{p-1}\left(\begin{array}{c}
-d_{p}(z) \\
e_{p}(z)
\end{array}\right) .
$$

For $\lambda \in \mathbb{R}$ denote by $C_{\lambda}$ the constant $J$-unitary matrix

$$
C_{\lambda}=\left\{\begin{array}{cc}
\left(\begin{array}{cc}
\lambda \alpha & \alpha \\
-\alpha^{-*}+\lambda \beta & \beta
\end{array}\right), & \alpha \neq 0, \\
\left(\begin{array}{cc}
\beta^{-*} & 0 \\
\lambda \beta & \beta
\end{array}\right), & \alpha=0 .
\end{array}\right.
$$

Then there exists a $\lambda$ such that

$$
\Theta(z):=\Theta_{1}(z) C_{\lambda}=\left(\begin{array}{cc}
a(z) & -b_{p-1} d_{p}(z) \\
c(z) & b_{p-1} e_{p}(z)
\end{array}\right),
$$

where $a$ and $c$ are polynomials such that $\operatorname{deg} c<\operatorname{deg} e_{p}=p$. The inclusion

$$
R_{0}\left(\begin{array}{l}
a \\
c
\end{array}\right) \in \mathcal{H}(\Theta)=\mathcal{H}\left(\Theta_{1}\right)=\mathcal{M}_{p}
$$

implies that $\operatorname{deg} a<p-1$. From $\operatorname{det} \Theta(z) \equiv 1$ it follows that $x=a$ and $y=c$ are polynomial solutions of the equation

$$
x(z) e_{p}(z)+y(z) d_{p}(z)=\frac{1}{b_{p-1}} .
$$

Since all polynomial solutions of this equation are given by

$$
x(z)=a(z)-s(z) d_{p}(z), \quad y(z)=c(z)+s(z) e_{p}(z)
$$

with some polynomial $s$, the solutions $x=a$ and $y=c$ have minimal degrees and because of that they are unique. Observing (4.15), we find

$$
a(z)=-d_{p-1}(z), \quad c(z)=e_{p-1}(z) .
$$

Hence

$$
\Theta(z)=\left(\begin{array}{cc}
-d_{p-1}(z) & -b_{p-1} d_{p}(z) \\
e_{p-1}(z) & b_{p-1} e_{p}(z)
\end{array}\right)=V(z)
$$

and $C_{\lambda}=\Theta(0)$ is the coefficient matrix of the fractional linear transformation (6.5). 
Define the function $\check{n}_{p}$ by (8.5). Then, according to Theorem 8.1] it is a Nevanlinna function. We show that $\check{n}_{p} \in \mathcal{L}\left(\check{n}_{p}\right)$. The function $f_{p}(\zeta)=\left(\begin{array}{ll}1 & -n(\zeta)) \mathbf{f}_{p}(\zeta)\end{array}\right.$ belongs to $\mathcal{L}(n)$ and, according to Theorem 8.1 (iii), it can be written as

$$
(1-n(\zeta)) \mathbf{f}_{p}(\zeta)=(1-n(\zeta)) \mathbf{u}_{p}(\zeta)+(a(\zeta)-n(\zeta) c(\zeta)) g_{p}(\zeta)
$$

with $\mathbf{u}_{p} \in \mathcal{M}_{p}, g_{p} \in \mathcal{L}\left(\check{n}_{p}\right)$, and the two summands on the righthand side are

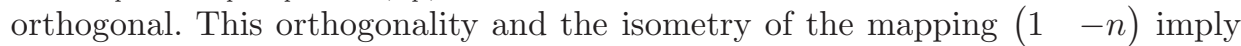
that $(0 \neq) \mathbf{f}_{p}-\mathbf{u}_{p} \in \mathcal{M}_{p}^{\perp}$ and hence there is a non-zero complex number $\gamma$ such that

Therefore

$$
\mathbf{f}_{p}-\mathbf{u}_{p}=\gamma\left(\begin{array}{c}
-d_{p}(\zeta) \\
e_{p}(\zeta)
\end{array}\right)
$$

$$
(a(\zeta)-n(\zeta) c(\zeta)) g_{p}(\zeta)=-\gamma\left(d_{p}(\zeta)+e_{p}(\zeta) n(\zeta)\right)
$$

and

$$
g_{p}(\zeta)=-\gamma \frac{e_{p}(\zeta) n(\zeta)+d_{p}(\zeta)}{-n(\zeta) c(\zeta)+a(\zeta)}=-\frac{\gamma}{b_{p-1}} \check{n}_{p}(\zeta)
$$

Hence $\check{n}_{p} \in \mathcal{L}\left(\check{n}_{p}\right)$. Equality (8.6) follows from item (3) in Section 3

\section{References}

[1] N.I. Akhiezer, The classical moment problem and some related topics in analysis, Fizmatgiz, Moscow, 1961; English transl., Hafner, New York, 1965.

[2] D. Alpay, R.W. Buursema, A. Dijksma, and H. Langer, The combined moment and interpolation problem for Nevanlinna functions, to appear.

[3] D. Alpay, A. Dijksma, and H. Langer, Factorization of J-unitary matrix polynomials on the line and a Schur algorithm for generalized Nevanlinna functions, Linear Algebra Appl. 387C (2004), 313-342,

[4] D. Alpay, A. Dijksma, H. Langer, and Y. Shondin, The Schur transform for generalized Nevanlinna functions: interpolation and self-adjoint operator realizations, Complex Analysis and Operator Theory 1 (2007), 189-120.

[5] D. Alpay, A. Dijksma, and H. Langer, The transformation of Issai Schur and related topics in an indefinite setting, Operator Theory: Adv., Appl., vol. 176, Birkhäuser Verlag, Basel, 2007, 1-98.

[6] D. Alpay and H. Dym, Structure invariant spaces of vector valued functions, Hermitian matrices and a generalization of the Iohvidov laws, Linear Algebra, Appl. $\mathbf{1 3 7 / 1 3 8}$ (1990) 137-181.

[7] L. de Branges, Some Hilbert spaces of analytic functions. I, Trans. Amer. Math. Soc. 106 (1963), 445-468.

[8] M. Derevyagin, On the Schur algorithm for indefinite moment problem, Methods of Functional Analysis and Topology 9(2) (2003), 133-145.

[9] A. Dijksma, H. Langer, A. Luger, and Y. Shondin, Minimal realizations of scalar generalized Nevanlinna functions related to their basic factorization, Operator Theory: Adv., Appl., 154, Birkhäuser Verlag, Basel, 2004, 69-90. 
[10] A. Dijksma, H. Langer, and H.S.V. de Snoo, Eigenvalues and pole functions of Hamiltonian systems with eigenvalue depending boundary conditions, Math. Nachr. 161 (1993), 107-154.

[11] H. Dym, J-contractive matrix functions, reproducing kernel Hilbert spaces and interpolation, CBMS Regional Conference Series in Mathematics 71, Amer. Math.Soc., Providence, RI, 1989.

[12] I.S. Kac and M.G. Krein, R-functions-analytic functions mapping the upper halfplane into itself, Amer. Math. Soc. Transl. 103(2) (1974), 1-18.

[13] M.G. Krein and H. Langer, Über einige Fortsetzungsprobleme, die eng mit der Theorie hermitescher Operatoren in Raume $\Pi_{\kappa}$ zusammenhängen. I. Einige Funktionenklassen und ihre Darstellungen, Math. Nachr. 77 (1977), 187-236.

[14] M.G. Krein and H. Langer, Über einige Fortsetzungsprobleme, die eng mit der Theorie hermitescher Operatoren in Raume $\Pi_{\kappa}$ zusammenhängen. II. Verallgemeinerte Resolvente, u-Resolventen und ganze Operatoren, J. Functional Analysis 30(3) (1978), 390-447.

[15] H. Langer and B. Textorius, On generalized resolvents and $Q$-functions of symmetric linear relations (subspaces), Pacific J. Math. 72 (1977), 135-165.

D. Alpay

Department of Mathematics

Ben-Gurion University of the Negev

P.O. Box 653

84105 Beer-Sheva, Israel

e-mail: dany@math.bgu.ac.il

A. Dijksma

Department of Mathematics

University of Groningen

P.O. Box 407

9700 AK Groningen, The Netherlands

e-mail: a.dijksma@math.rug.nl

H. Langer

Institute of Analysis and Scientific Computation

Vienna University of Technology

Wiedner Hauptstrasse 8-10

A-1040 Vienna, Austria

e-mail: hlanger@mail.zserv.tuwien.ac .at 